\title{
Estimation of Delay Times in Biological Systems
}

\author{
T. MÜller, ${ }^{1,2}$ M. Lauk, ${ }^{1,2}$ M. Reinhard, ${ }^{3}$ A. Hetzel, ${ }^{3}$ C. H. LÜCKInG, ${ }^{3}$ and J. Timmer ${ }^{1,2}$ \\ ${ }^{1}$ Center for Data Analysis and Modeling, University of Freiburg, Eckerstrasse 1, 79104 Freiburg, Germany; ${ }^{2}$ Department of Physics, \\ University of Freiburg, Hermann-Herder-Strasse 3, 79104 Freiburg, Germany; and ${ }^{3}$ Department of Neurology and Clinical \\ Neurophysiology, University of Freiburg, Breisacherstrasse 64, 79106 Freiburg, Germany
}

\begin{abstract}
The problem of delay time estimation in biological systems is addressed with the focus on practical applicability of methods. Four delay time estimators are described: a cross correlation method and three increasingly sophisticated interpretations of the phase spectrum, ranging from a pointwise interpretation of the phase spectrum in terms of a delay to a Hilbert transform method. The four methods are compared through simulation studies showing that, in general, the Hilbert transform method performs best. The methods are then used to estimate delay times in three physiological systems: vestibular stimulation, cerebral autoregulation, and human orthostatic tremor. In all three cases, the Hilbert transform method yields the best results, leading in some cases to physiologically more sensible interpretations of experiments than the other methods.
\end{abstract}

Keywords-Cross-spectral analysis, Hilbert transform, Vestibular stimulation, Cerebral autoregulation, Orthostatic tremor.

\section{INTRODUCTION}

The analysis of time series measured from biological systems poses many methodological challenges. This article addresses the question of delay time estimation from multivariate biological data. Knowing the delay time between two signals can often help to understand the physiology of a given system, e.g., the delay time can indicate whether a given neural impulse has traveled via a fast reflex loop or via the central nervous system. ${ }^{30}$

The estimation of delay times is not straightforward. Often the measured system does not just transmit the signal between input and output with a pure time delay, but acts as a filter, while no validated parametric model of the system is available. Thus, one has to use a nonparametric method to estimate the delay time. The literature on delay time estimation is large. , $3,6-8,10-12,14-21,26,31,32,36,47,48$ The proposed methods stem mostly from linear system analysis. Most biological systems exhibit some degree of nonlinearity, making it

Address correspondence to J. Timmer, Center for Data Analysis and Modeling, University of Freiburg, Eckerstr. 1, 79104 Freiburg, Germany. Electronic mail: jeti@fdm.uni-freiburg.de uncertain whether methods developed for the analysis of linear systems are applicable. Delay times in biological systems could also be frequency dependent. Thus the choice of a procedure for delay time estimation in biological systems is a methodological challenge.

In this article, we compare four methods for delay time estimation: (i) Interpreting the lag at which the cross correlation between input and output is maximal as a delay time, (ii) interpreting the phase spectrum between input and output at the frequency with maximum coherency in terms of a delay time, (iii) interpreting the phase spectrum in terms of a delay time by fitting a straight line whose slope gives an estimate of the delay, and (iv) a Hilbert transform method that accounts for the filter properties of the system by correcting the phase spectrum before fitting a straight line to it. Although the four proposed methods require no explicit model of the measured system, they are all based on a a priori assumptions about the structure of the measured system: (i) -(iii) assume a "delay only" model, while (iv) only assumes that the system between input and output has the so-called minimum phase property. Thus from a theoretical point of view it is relatively easy to assess the merits of the four methods. However, our aim is to assess their performance from the point of view of a data analyst who possesses no validated information about the measured system. It is quite certain that some of the assumptions of all the four models are violated to some degree by empirical systems. The important question for us is how the methods perform under such circumstances. We compare the methods using simulation studies with five different models: a damped stochastic linear oscillator, a stochastic linear oscillator with nonlinear input, a nonlinear threshold system, a linear low-pass filter, and a linear high-pass filter. The four methods are then applied to three measured biological data sets taken from experiments on vestibular stimulation, cerebral autoregulation, and human tremor.

The article is organized as follows: The Methods section gives some mathematical background on crossspectral analysis and describes the four proposed delay 
estimation methods. Simulations introduces the simulation methods used to compare the estimators and reports the results of our simulations. Applications employs the four proposed methods for the analysis of measured data from three different biological systems for which delay time estimation is important for understanding the physiology. Finally, in the Discussion section, the results of our analyses of the measured biological data are discussed in light of the results from the simulation studies.

\section{METHODS}

In this section, we give the mathematical background for the delay time estimators that will be compared in this article. We first outline some methods of crossspectral analysis and then describe the four delay time estimators that we will use in the rest of the article.

\section{Cross-Spectral Analysis}

Mathematical Background. The power spectrum (or power spectral density) $S_{X}(f)$ of a stationary, zero mean, time discrete process $X(t), t \in \mathcal{Z}$, is defined as the Fourier transform (FT) of the autocorrelation function $\mathrm{AC}_{X}(\tau)$ :

$$
\begin{gathered}
\mathrm{AC}_{X}(\tau)=\langle X(t) X(t-\tau)\rangle, \quad \tau \in \mathcal{Z}, \\
S_{X}(f)=\sum_{\tau=-\infty}^{\infty} \mathrm{AC}_{X}(\tau) \exp (-2 \pi i f \tau),
\end{gathered}
$$

where " \langle\rangle " denotes expectation and $\mathcal{Z}$ is the set of integers. For Eq. (2) to be well-defined, one has to assume that $\mathrm{AC}_{X}$ decays fast enough at large lags (Proposition 10.3.1 in Ref. 5).

Analogously to the univariate case, the cross spectrum $S_{X Y}(f)$ of two stationary, zero mean, time discrete processes $X(t)$ and $Y(t)$ is defined as the Fourier transform of the cross-correlation function $\mathrm{CC}_{X Y}(\tau)=\langle X(t) Y(t$ $-\tau)\rangle$ (Definition 11.6.1. in Ref. 5):

$$
\begin{aligned}
S_{X Y}(f) & =\frac{1}{2 \pi} \sum_{\tau=-\infty}^{\infty} \mathrm{CC}_{X Y}(\tau) \exp (-2 \pi i f \tau) \\
& =\left\langle\widetilde{X}(f) \tilde{Y}^{*}(f)\right\rangle,
\end{aligned}
$$

where * denotes complex conjugation and $\tilde{X}(f)$ and $\tilde{Y}(f)$ are the normalized discrete Fourier transforms of $X(t)$ and $Y(t)$, respectively.

The coherency spectrum $\operatorname{coh}_{X Y}(f)$ is defined as the normalized modulus of the cross spectrum $S_{X Y}(f)$ :

$$
\operatorname{coh}_{X Y}(f)=\frac{\left|S_{X Y}(f)\right|}{\sqrt{S_{X}(f) S_{Y}(f)}},
$$

where $S_{X}(f)$ and $S_{Y}(f)$ denote the power spectra of $X(t)$ and $Y(t)$, respectively. The coherency is a measure for linear predictability: if $X$ and $Y$ are linearly related, $\operatorname{coh}_{X Y}$ is equal to its maximum value 1 ; if there is no linear relationship between $X$ and $Y, \operatorname{coh}_{X Y}$ is equal to its minimum value 0 .

The gain describes the frequency-dependent amplitude transmission from input to output:

$$
\mathrm{G}_{X Y}(f)=\frac{\left|S_{X Y}(f)\right|}{S_{X}(f)} .
$$

The phase spectrum $\Phi_{X Y}(f)$ is defined, up to a modulus of $2 \pi$, by the representation

$$
S_{X Y}(f)=\left|S_{X Y}(f)\right| \exp \left[i \Phi_{X Y}(f)\right]
$$

It is the subject of spectral analysis to estimate these quantities from measured data.

Cross-Spectral Analysis of Measured Data. Empirical data are usually measured (sampled) at discrete times $t_{i}=i \Delta t, \quad i=0, \ldots, N-1$. The estimation of spectra from given data is confronted with two main problems, variance and leakage. The problem of variance is that the straightforward estimator of the power spectrum, the socalled periodogram [Eq. (11)], is not consistent: with increasing amount of data, the variance of this power spectrum estimator at a given frequency does not decrease. Leakage is an effect arising from the convolution theorem: the Fourier transform of a finite stretch of data is the convolution of the Fourier transform of the underlying process and the Fourier transform of a window function that selects the finite stretch of data. As this window has sharp edges, its Fourier transform shows slowly decreasing sidebands, thus transferring power from a peak to adjacent frequency bins. There are wellestablished methods of spectral estimation available for dealing with these two issues. ${ }^{4,5,38}$

The procedure used here is as follows: Two given time series $x\left(t_{i}\right), y\left(t_{i}\right), i=0, \ldots, N-1$, sampled with sampling interval $\Delta t$, are first tapered with a window function $W_{\text {tap }}(i)$ rising from zero to unity and falling back to zero again:

$$
x_{\text {tap }}\left(t_{i}\right)=x\left(t_{i}\right) \cdot W_{\text {tap }}(i), \quad i=0, \ldots, N-1 .
$$

This reduces the problem of leakage if one uses a window with smooth edges. We use a triangular window, the so-called Bartlett window: 


$$
W_{\text {tap }}(i)=1-\frac{|(N-1) / 2-i|}{(N-1) / 2}, \quad i=0, \ldots, N-1 .
$$

Then the discrete Fourier transform of the tapered data

$$
\widetilde{x}(f)=\frac{1}{\sqrt{N}} \sum_{j=0}^{N-1} x_{\text {tap }}\left(t_{j}\right) \exp \left(-2 \pi i f t_{j}\right)
$$

is computed via the FFT algorithm. ${ }^{37}$ In what follows, we assume $N$ even for ease of notation. The discrete FT is evaluated at frequencies

$$
f_{j}=\frac{j}{N \Delta t}, \quad j=0, \ldots, N / 2,
$$

i.e., from $f_{0}=0$ to the Nyquist frequency $f_{\mathrm{Nyq}}=f_{N / 2}$ $=2 / \Delta t$, which is the maximum frequency that can be detected using a sampling interval of $\Delta t$. The frequency resolution is $1 /(N \Delta t)$, i.e., the longer the measurement, the finer the frequency resolution.

From the discrete FTs $\tilde{x}$ and $\tilde{y}$ of the tapered data, the periodograms $P_{x}$ and $P_{y}$ are calculated:

$$
P_{x}\left(f_{j}\right)=\left|\widetilde{x}\left(f_{j}\right)\right|^{2}, \quad P_{y}\left(f_{j}\right)=\left|\widetilde{y}\left(f_{j}\right)\right|^{2} .
$$

While the expectation value $\left\langle P_{x}\left(f_{j}\right)\right\rangle$ of the periodogram is the power spectrum $S_{x}\left(f_{j}\right)$, the periodogram is not a consistent estimator for the spectrum, since its variance does not decrease with increasing amount of data, with increasing $N$, only the frequency resolution becomes finer. One can however trade frequency resolution for variance, and there are a number of ways to do this. Our procedure is to smooth the periodogram with a smoothing window $W_{S}(k), k=-h, \ldots, h$. The window width $2 h+1$ must be chosen such as to yield a good compromise between bias and variance: the larger $h$, the smaller the variance of the estimate, but the larger the bias. We use a triangular window normalized to sum to unity:

$$
W_{S}(k)=\frac{1}{h}-\frac{|k|}{h^{2}}, k=-h, \ldots, h,
$$

with $h$ chosen such as to yield a sufficient frequency resolution. (There are adaptive algorithms for choosing the window width as well. ${ }^{42}$ ) Our estimator $\hat{S}_{x}\left(f_{j}\right)$ for the spectrum $S_{x}\left(f_{j}\right)$ at frequency $f_{j}$ is therefore

$$
\hat{S}_{x}\left(f_{j}\right)=\sum_{k=-h}^{h} P_{x}\left(f_{j+k}\right) \cdot W_{S}(k) .
$$

The estimates $\hat{S}_{y}$ and $\hat{S}_{x y}$ are defined exactly analogously, and estimates for coherency, gain, and phase are obtained by plugging these estimates into the respective defining Eqs. (4), (5), and (6) (Chap. 11 in Ref. 5). The variance of the estimated phase spectrum is approximated by

$$
\operatorname{var}\left[\hat{\Phi}_{x y}\left(f_{j}\right)\right] \approx \frac{1}{\nu}\left(\frac{1}{\operatorname{coh}_{x y}^{2}\left(f_{j}\right)}-1\right),
$$

where the effective number of degrees of freedom, $\nu$, depends on the taper window and the smoothing window used (Sec. 8.5 in Ref. 4):

$$
\begin{gathered}
\nu=\frac{2 q_{2}^{2}}{q_{4}}\left(\sum_{k=-h}^{h} W_{S}^{2}(k)\right)^{-1}, q_{2}=\frac{1}{N} \sum_{t=0}^{N-1} W_{\text {tap }}^{2}(t), \\
q_{4}=\frac{1}{N} \sum_{t=0}^{N-1} W_{\text {tap }}^{4}(t) .
\end{gathered}
$$

In Eq. (14), the variance of the phase spectrum is monotonically related to the inverse of the squared coherency. The approximation of Eq. (14) only holds if, compared to $\pi^{2}$, the variance is small (Ref. 4).

At significance level $\alpha$, the value

$$
s=\sqrt{1-\alpha^{2 /(\nu-2)}}
$$

gives the threshold below which the null hypothesis of zero coherency cannot be rejected. Under this null hypothesis, the phase spectrum is uniformly distributed on the interval $[-\pi, \pi]$ (Ref. 5).

Stationarity and Linearity. Data from biological systems are hardly ever stationary. However, cross-spectral analysis can be applied fruitfully to such systems as long as the underlying input-output relation is time invariant, which is often the case even outside a system's steady state. Cross-spectral methods are based on the assumption of a linear input-output relation. This assumption is also generally violated to some extent in real life systems. We explore the limits of the linearity assumption via simulation studies.

Influence of Observational Noise. Observational noise influences the phase spectrum only indirectly. Nevertheless, noise is the main reason for some problems discussed in this article. If the output $y(t)$ is linearly related to the input $x(t)$, the coherency should be unity. However, if observational white noise is added to the output, the coherency is determined by the ratio of the variance $\sigma^{2}$ of the noise and the spectrum $S_{y}(f)$ of $y(t)$. Within 
frequency bands where the power of the spectrum $S_{y}(f)$ is small compared to the variance of the observational noise, the coherency approaches zero. Consequently, the phase spectrum in these frequency bands corresponds to a uniformly distributed random variable and cannot be used for further analysis. This often leads to the situation that the frequency band where the coherency estimate is significant is rather small, although the underlying processes might be coherent over a broad band. These considerations can be generalized to coherency estimates for two linearly related processes both covered by observational noise. ${ }^{43}$

\section{Delay Time Estimation}

Cross-spectral analysis is a key ingredient in most procedures for time delay estimation. We present four relatively simple estimators: using the lag with maximum cross correlation as a delay estimate, interpreting the phase spectrum at a single frequency in terms of a delay, fitting a straight line to the phase spectrum, and a Hilbert transform method. Apart from these estimators, parametric models should of course be considered whenever appropriate. However, in this article we assume that a delay time is to be estimated in a system for which no validated parametric model is known. Note that the popular parametric ARMA models (Chap. 3 in Ref. 5) are largely covered by the Hilbert transform method.

In the following, we assume that data $x\left(t_{i}\right)$ ("input") and $y\left(t_{i}\right)$ ("output") are sampled from two stationary processes at times $t_{i}=i \Delta t, i=0, \ldots, N-1$.

Using the Maximum of the Cross Correlation. Assuming that $y(t)$ is a time-shifted copy of $x(t)$, i.e., $y(t+\delta)$ $=x(t)$, the delay $\delta$ can be estimated as the lag at which the estimated cross correlation between input and output is maximal:

$$
\hat{\delta}=\max _{\tau}\left|\widehat{C C}_{x y}(\tau)\right|
$$

Under certain assumptions, Eq. (17) is an unbiased estimate for the delay time $\delta^{19}$ [see Figs. $9(\mathrm{~F})$ and $10(\mathrm{~F})$ for an illustration of the cross correlation function estimated from empirical data sets].

Pointwise Interpretation of the Phase Spectrum. This method, like the following two, is based on an analysis of the phase spectrum between input and output. In general, for every linear system

$$
y(t)=\int_{-\infty}^{\infty} d \tau \mathrm{a}_{x y}(\tau) x(t-\tau)=\left(\mathrm{a}_{x y} \star x\right)(t),
$$

the system's impulse response function $\mathrm{a}_{x y}(\tau)$ can be written as the convolution of three subsystems with specific properties:

$$
\mathrm{a}_{x y}(\tau)=\left(\operatorname{delay}_{\delta} \star \mathrm{mp} \star \mathrm{ap}\right)(\tau),
$$

where the first component is a pure delay, mp is a socalled minimal phase system, and ap is an all-pass filter. The phase spectrum $\Phi_{x y}(f)$ is determined solely by the function $\mathrm{a}_{x y}$, since by Eq. (3) and Eq. (18),

$$
S_{x y}(f)=\widetilde{\mathrm{a}}_{x y}^{*}(f) S_{x}(f),
$$

and as $S_{x}(f)$ is real,

$$
\Phi_{x y}(f)=\arg S_{x y}(f)=\arg \widetilde{\mathrm{a}}_{x y}^{*}(f) .
$$

If the system consists of a delay only, $\mathrm{a}_{x y}(\tau)$ is a delta distribution at lag $\delta$. The Fourier transform of this transfer function is

$$
\widetilde{\mathrm{a}}_{x y}(f)=\exp (-2 \pi i f \delta)
$$

from which it follows that the phase spectrum is a straight line through the origin with the slope given by the delay time $\delta$ :

$$
\Phi_{x y}(f)=2 \pi f \delta
$$

In the more general case where the transfer function has the form of Eq. (19), the phase spectrum is given by

$$
\Phi_{x y}(f)=2 \pi f \delta+\arg \widetilde{m p}^{*}(f)+\arg \widetilde{\operatorname{ap}}^{*}(f) .
$$

In the pointwise interpretation method, one assumes a delay-only model and uses Eq. (23) at a single frequency to infer a delay time between input and output. From Eq. (14) it is clear that the frequency at which the coherency is maximal will yield the smallest error for the delay time estimate. Consequently, we use that frequency in the estimate:

$$
\begin{gathered}
\hat{\delta}=\hat{\Phi}_{x y}\left(f_{c}\right) / 2 \pi f_{c}, \quad \text { where } f_{c}=\max _{f} \widehat{\operatorname{coh}}_{x y}(f) \\
\text { and } \hat{\Phi}_{x y}\left(f_{c}\right) \in(-\pi, \pi] .
\end{gathered}
$$

Fitting a Straight Line to the Phase Spectrum. A pointwise interpretation of the phase spectrum is certainly suboptimal even if the delay-only model holds, since it ignores the information present in the rest of the phase 
spectrum. That information can be used by fitting a straight line to a part of the phase spectrum that shows significant coherency.

The fit can be achieved by a generic least-squares or weighted least-squares procedure giving a maximum likelihood estimate. ${ }^{37}$ Applying such a procedure is hampered by the fact that the phase estimate can show discontinuities as it is only defined up to a modulus of $2 \pi$. One remedy for this problem is to use so-called phase unwrapping algorithms that produce a continuous phase spectrum. ${ }^{45}$ However, such algorithms are generally not robust in the case of a low signal-to-noise ratio. A better approach is to use the cosine of the residuals. The cosine function is locally quadratic and automatically takes care of the periodicity problem. ${ }^{14}$ Weights are chosen to reflect the variance of the phase spectrum estimate [Eq. (14)]. Thus, one defines the objective function

$$
\operatorname{obj}(\delta)=\sum_{f_{j} \in B} \frac{\widehat{\operatorname{coh}^{2}}\left(f_{j}\right)}{1-\widehat{\operatorname{coh}}^{2}\left(f_{j}\right)} \cos \left[\hat{\Phi}_{x y}\left(f_{j}\right)-2 \pi f_{j} \delta\right],
$$

where $B$ is either the full range of available frequencies with significant coherency or a subset thereof. This makes it possible, e.g., to restrict the fitting to physiologically important frequencies for a given application. The delay time estimate is given by the delay at which the objective function is maximal:

$$
\hat{\delta}=\max _{\delta} \operatorname{obj}(\delta)
$$

It can be shown that this estimate asymptotically approaches the weighed least-squares maximum likelihood method, ${ }^{14,17}$ [see Figs. 9(E) and 10(E) for an illustration of the objective function estimated from empirical data sets].

Hilbert Transform Method. Looking back at Eq. (24), it is clear that one could improve on the method of the previous section if one could somehow correct the phase spectrum before fitting a straight line to it. If one could subtract the contributions of the minimum phase system and the all-pass system, the delay-only model would definitely hold, and the fitting procedure just described could be applied to linear systems without any reservations.

It is generally not possible to correct the phase spectrum for both the minimum phase contribution and the all-pass contribution. However, many physical systems can be modeled as minimum phase systems without an all-pass component, e.g., as AR models. The Hilbert transform relation links the phase of the transfer function of a minimum phase system to the logarithm of the system's gain. This relation allows for subtracting the contribution of the minimum phase component from Eq. (24). Note that this application of the Hilbert transform is different from the usual application of this transform in system analysis, which is signal demodulation (see Chap. 7.3 in Ref. 33).

The Hilbert transform relation for a minimum phase system asserts that, see Eq. (7.22) in Chap. 7.2 in Ref. 33:

$$
\begin{aligned}
\arg \widetilde{\mathrm{mp}}_{x y}\left(f_{j}\right)= & \frac{1}{N} \sum_{k=1, k \neq j}^{N / 2} \log \mathrm{G}_{x y}\left(f_{k}\right)\left(\cot \frac{2 \pi\left(f_{j}-f_{k}\right)}{2}\right. \\
& \left.+\cot \frac{2 \pi\left(f_{j}+f_{k}\right)}{2}\right) .
\end{aligned}
$$

$\mathrm{G}_{x y}(f)$ denotes the gain of the minimum phase system. However, the presence of a delay or even of an all-pass filter does not influence the gain, since both a pure delay and an all-pass have constant gain unity. Thus the gain of the minimal phase system is equal to the gain of the total system, which can be estimated from the data [cf. Eq. (5)]. This fact allows for an estimate $\widehat{\arg \widetilde{m p}_{x y}}\left(f_{j}\right)$ of the minimal phase using Eq. (28) directly. By the symmetry properties of the cotangent function, the estimate is independent of any constant scaling factor in $\mathrm{G}_{x y}(f)$. This feature is important, since in an application where input and output are different physical quantities, the choice of units introduces an arbitrary constant scaling in the gain.

Analogously to Eqs. (26) and (27), an objective function is defined and maximized to find an estimate $\hat{\delta}$ for the delay time $\delta:^{31}$

$$
\begin{gathered}
\operatorname{obj}(\delta)=\sum_{f_{j} \in B} \frac{\widehat{\operatorname{coh}^{2}}\left(f_{j}\right)}{1-\widehat{\operatorname{coh}}^{2}\left(f_{j}\right)} \cos \left[\hat{\Phi}_{x y}\left(f_{j}\right)-\widehat{\arg \widetilde{\mathrm{mp}}_{x y}}\left(f_{j}\right)\right. \\
\left.-2 \pi f_{j} \delta\right], \\
\hat{\delta}=\max _{\delta} \operatorname{obj}(\delta) .
\end{gathered}
$$

The frequency band $B$ can again be chosen to reflect $a$ priori information about the system under consideration. As the Hilbert transform method relaxes the assumption of a simple delay-only model, it is the most general method proposed here, but it still assumes that the system is linear and that no all-pass filter is active in the system [see Figs. 9(D) and 10(D) for an illustration of the objective function estimated from empirical data sets]. 


\section{SIMULATIONS}

In this section, the four delay estimators described above are compared via simulation studies. These simulation studies allow us to assess the influence of observational noise, of nonlinear influences, and of various model types. Furthermore, as the data from our simulated systems are similar to the measured data analyzed in the next section, the simulations also give important hints as to how the four proposed delay estimators perform in the case of measured data.

We first describe five models used in our simulations and then present the results of our simulations. All cross spectra were estimated after tapering the data with a triangular window [Eq. (7)] by smoothing the periodograms with a window of $h=100$ frequency bins [Eq. (13)].

\section{Systems used for Simulation}

We investigate the performance of the four estimators using simulated data from five systems: three stochastic oscillators, a linear low-pass filter, and a linear high-pass filter. Data are simulated with a virtual sampling frequency of $100 \mathrm{~Hz}$ for ease of comparison with empirical data. Since empirical data are usually affected by additive observational noise, we added observational noise with a signal-to-noise ratio [Eq. (37)] of 1 to all simulated data sets.

Stochastic Linear Oscillator. As a damped stochastic linear oscillator we choose an autoregressive process of order two (AR[2] process) $y(t)$ driven by Gaussian white noise with mean zero and unit variance, $x(t)$ $\sim W N(0,1)$. Explicitly, the model is

$$
y\left(t_{j}\right)=x\left(t_{j}-\delta\right)+a_{1} y\left(t_{j-1}\right)+a_{2} y\left(t_{j-2}\right),
$$

where $\delta$ is the delay time. The parameters $a_{1}$ and $a_{2}$ are linked to the period $T$ and relaxation time $\tau$ of the oscillator as follows: ${ }^{22}$

$$
a_{1}=2 \cos \left(\frac{2 \pi}{T}\right) \exp \left(\frac{-1}{\tau}\right), \quad a_{2}=-\exp \left(\frac{-2}{\tau}\right)
$$

Parameters used were $a_{1}=1.96907$ and $a_{2}=$ -0.97531 , corresponding to $T=0.8 \mathrm{~s}$ and $\tau=0.8 \mathrm{~s}$.

Figure 1 shows the result of cross-spectral analysis of one realization of the $\mathrm{AR}[2]$ process.

Stochastic Oscillator with Nonlinear Input. In the previous section, the input $x(t)$ of the stochastic oscillator [Eq. (31)] was Gaussian white noise. In this section we investigate the performance of the delay estimators in the case of a stochastic oscillator driven by a "nonlinear" input: while we retain the $\mathrm{AR}[2]$ model of Eq. (31), we use as input $x$ the $x_{1}$ (location) component of a stochastic nonlinear van der Pol oscillator ${ }^{46}$ driven by Gaussian white noise $\epsilon(t) \sim W N\left(0, \sigma^{2}\right)$ :

$$
\begin{gathered}
\dot{x}_{1}(t)=x_{2}(t), \\
\dot{x}_{2}(t)=\mu\left[1-x_{1}(t)^{2}\right] x_{2}(t)-x_{1}(t)+\epsilon(t) .
\end{gathered}
$$

Equation (33) is integrated using the lowest-order Euler integration scheme with step size $h$ due to the presence of the noise term $\epsilon^{25,41}$ For our simulations, the parameters for the van der Pol oscillator are $\mu=2, \sigma^{2}=1$, and $h=0.1$ time steps $(h=1 \mathrm{~ms})$, leading to a peak at 15 Hz. Parameters for the $\mathrm{AR}[2]$ process are again $a_{1}$ $=1.96907$ and $a_{2}=-0.97531$, corresponding to $T$ $=0.8 \mathrm{~s}$ and $\tau=0.8 \mathrm{~s}$.

Figure 2 shows the result of cross-spectral analysis of input $\left[x=x_{1}\right.$ component of Eq. (33) $]$ and output $[y$ component of the AR[2] process, Eq. (31)] for one realization. Note the low coherency everywhere except around the peak frequency of the input oscillator, $\sim 15 \mathrm{~Hz}$, which accounts for the poor performance of crossspectral methods for this particular model (cf. Table 1).

Stochastic Oscillator with Nonlinear Transfer Function. In this section we study a system that violates the assumption of a linear transfer function between input and output. This assumption underlies all four proposed delay estimators. It is important to study the nonlinear case since biological systems typically exhibit some degree of nonlinearity.

As a nonlinear stochastic oscillator, we choose a threshold autoregressive process of order two (SETAR[2]). ${ }^{44}$ This process is derived from the AR[2] process studied so far by introducing a component that is sensitive to a threshold $s$ :

$$
\begin{aligned}
y\left(t_{j}\right)= & x\left(t_{j}-\delta\right)+a_{1} y\left(t_{j-1}\right) \\
& + \begin{cases}a_{2}^{+} y\left(t_{j-2}\right) & \text { if } y\left(t_{j-2}\right)>s, \\
a_{2}^{-} y\left(t_{j-2}\right) & \text { if } y\left(t_{j-2}\right) \leqslant s .\end{cases}
\end{aligned}
$$

The driving input $x(t)$ is Gaussian white noise: $x(t)$ $\sim \mathrm{WN}(0,1)$. Parameters used were $a_{1}=1.6, a_{2}^{+}=-2.3$, $a_{2}^{-}=-0.72$, and $s=2.5$.

Figure 3 shows the result of cross-spectral analysis of input and output for one realization. The second peak in the power spectrum [Fig. 3(A)] at double the fundamental frequency is a higher harmonic, which is typical for nonlinear systems. 
A
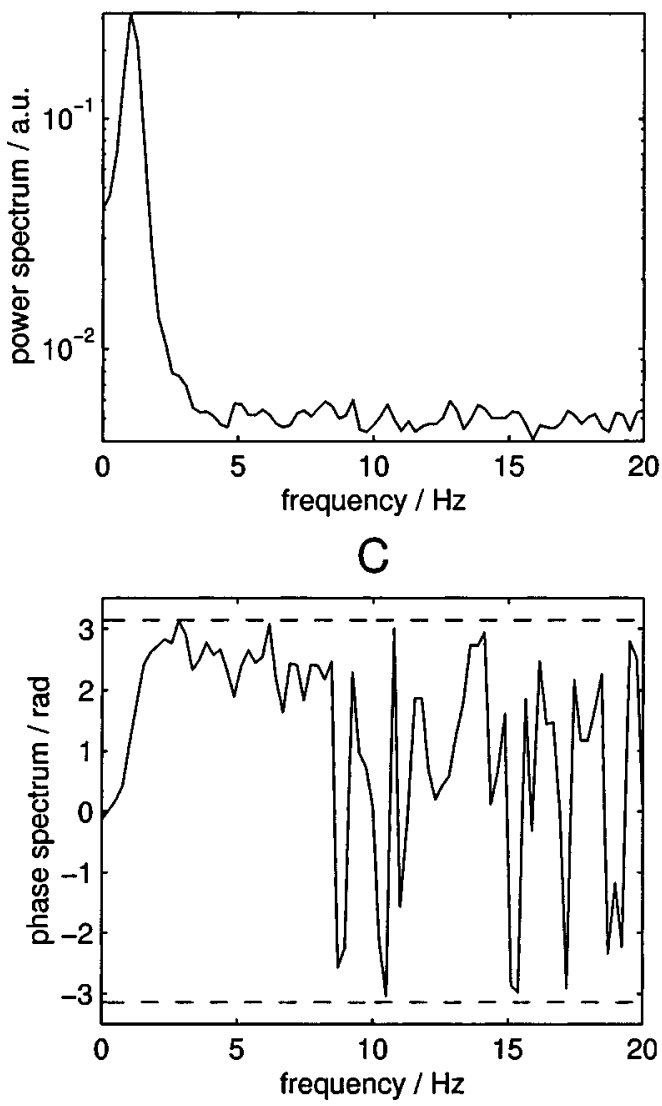

B
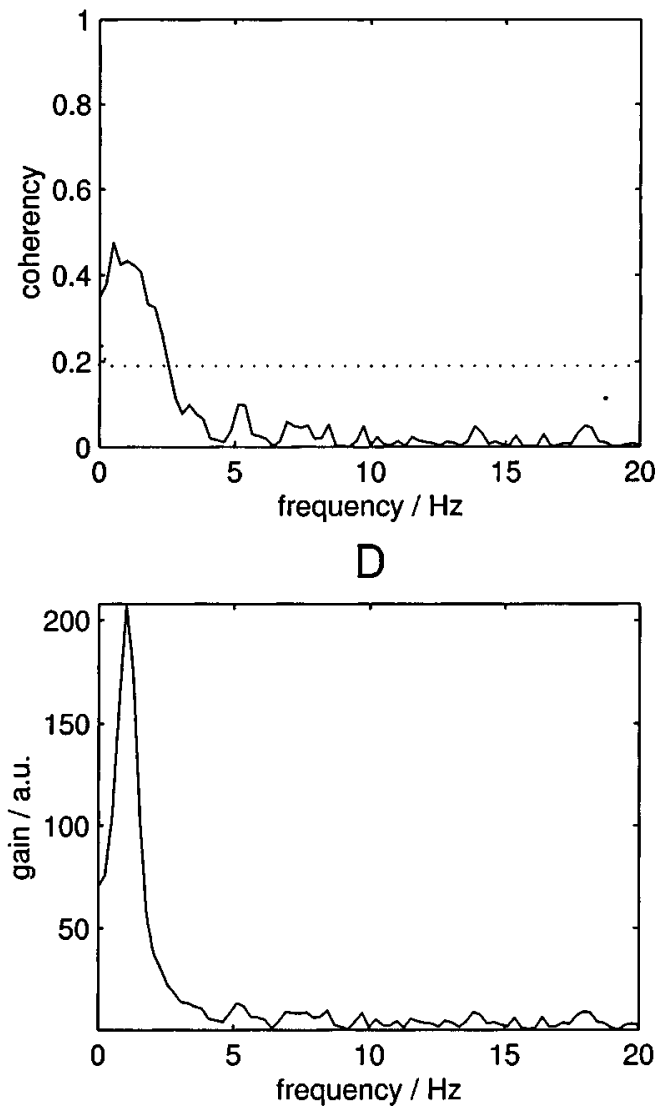

FIGURE 1. Cross-spectral analysis of input and output for one realization of the linear stochastic oscillator with Gaussian white noise as input. (A) Power spectrum of output; (B) coherency (significance threshold, $\alpha=0.05$, indicated by dotted line); (C) phase spectrum; (D) gain. Noise level, $S N R_{\text {in }}=S N R_{\text {out }}=1$. No delay. Parameters: $a_{1}=1.96907, a_{2}=-0.97531$.

Linear Low-Pass Filter. A simple linear low-pass filter is given by a moving average process of order four $(\mathrm{MA}[4]$ process):

$$
\begin{aligned}
y\left(t_{j}\right)= & m_{-2} x\left(t_{j-2}\right)+m_{-1} x\left(t_{j-1}\right)+m_{0} x\left(t_{j}\right)+m_{1} x\left(t_{j+1}\right) \\
& +m_{2} x\left(t_{j+2}\right)
\end{aligned}
$$

with parameters $m_{-2}=m_{2}=7 / 96, m_{-1}=m_{1}=1 / 4$, and $m_{0}=17 / 48$. The input $x(t)$ is Gaussian white noise of unit variance, $x(t) \sim \mathrm{WN}(0,1)$.

Figure 4 shows the result of cross-spectral analysis of input and output for one realization, exhibiting the lowpass characteristic in the gain function. The true phase spectrum is zero due to the symmetric form of Eq. (35).

Linear High-Pass Filter. A simple linear high-pass filter can also be implemented as an MA[4] process [Eq. (35)] with parameters $m_{-2}=m_{2}=-7 / 96, m_{-1}=m_{1}=-1 / 4$, and $m_{0}=31 / 48$. The input $x(t)$ is again Gaussian white noise of unit variance, $x(t) \sim \mathrm{WN}(0,1)$.
The result of cross-spectral analysis looks like for the low-pass filter, but with high and low frequencies reversed (not shown).

\section{Simulation Results}

Influence of Observation Noise. The influence of observational noise was assessed using the AR[2] model [Eq. (31)]. The data $x_{\text {in }}(t)$ and $y_{\text {out }}(t)$ used for delay estimation were derived from the series $x(t)$ and $y(t)$ by adding uncorrelated Gaussian white noise $\epsilon_{\text {in }}, \epsilon_{\text {out }}$ $\sim \mathrm{WN}(0,1)$ :

$$
x_{\text {in }}(t)=x(t)+c_{\text {in }} \epsilon_{\text {in }}(t), \quad y_{\text {out }}(t)=y(t)+c_{\text {out }} \epsilon_{\text {out }}(t) \text {. }
$$

We present our results in terms of the signal-to-noise ratio (SNR), which is related to the constants $c_{\text {in }}$ and $c_{\text {out }}$ via 
A

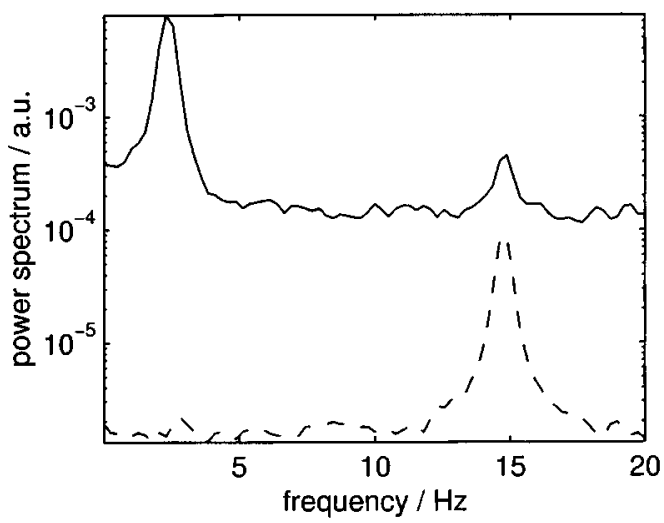

$\mathrm{C}$

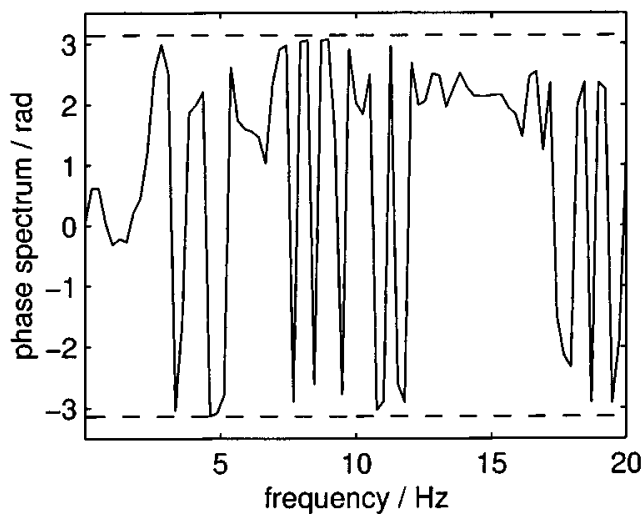

B
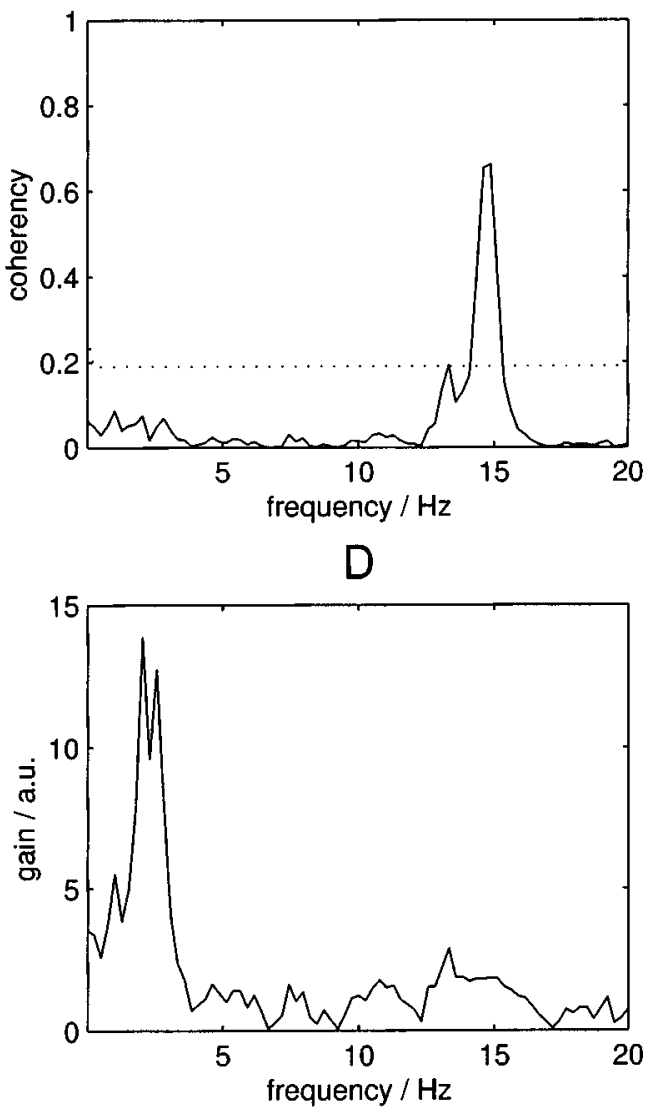

FIGURE 2. Cross-spectral analysis of input and output for one realization of the stochastic oscillator driven by a nonlinear input. (A) Power spectrum of input (dashed) and output (solid line); (B) coherency (significance threshold, $\alpha=0.05$, indicated by dotted line); (C) phase spectrum; (D) gain. Noise level, $S_{N R} R_{\text {in }}=S_{N R}$ out $=1$. No delay. Parameters: $\mu=2, \sigma^{2}=1, h=0.1$ bins, $a_{1}$ $=1.96907$, and $a_{2}=-0.97531$.

$$
\mathrm{SNR}_{\mathrm{in}}=\frac{\operatorname{variance}(\text { input signal })}{\operatorname{variance}(\text { noise })}=\frac{\operatorname{var}[x(t)]}{c_{\text {in }}},
$$

analogously for $\mathrm{SNR}_{\text {out }}$. We use SNRs from 0.5 (noise twice as strong as the signal) to infinity (no noise added), which well covers the range of noise levels found in our applications. For each noise level, 100 realizations of the $\mathrm{AR}[2]$ process, each of length $2^{15}$ samples, are simulated. In order to facilitate comparison with the data section, we assume a virtual sampling frequency of 100 $\mathrm{Hz}$, which means that the length of our simulated data sets corresponds to $\sim 5 \frac{1}{2}$ min. Figure 5 shows the results of our simulations.

As can be seen, the performance of the estimators depends on both $\mathrm{SNR}_{\text {in }}$ and $\mathrm{SNR}_{\text {out }}$. The bias of the estimate is most strongly influenced by $\mathrm{SNR}_{\text {out }}$, while the variance of the estimate is more strongly influenced by $\mathrm{SNR}_{\text {in }}$. The "single frequency" method performs

TABLE 1. Comparison of the four delay estimators using the five models. Numbers given are estimated delays \pm standard deviation in seconds from 100 trials. Signal-to-noise ratio, $\mathrm{SNR}_{\text {in }}=\mathrm{SNR}_{\text {out }}=1$. True delay, $0.2 \mathrm{~s}$. Method names abbreviated in column headings.

\begin{tabular}{lcccc}
\hline Model & Max. CC & Single freq. & Line fit & Hilbert meth. \\
\hline AR[2] & $0.37 \pm 0.02$ & $0.30 \pm 0.14$ & $0.41 \pm 0.01$ & $0.24 \pm 0.01$ \\
AR[2] nonlin. input & $0.27 \pm 0.03$ & $0.02 \pm 0.01$ & $0.08 \pm 0.04$ & $0.07 \pm 0.09$ \\
SETAR[2] & $0.27 \pm 0.01$ & $0.01 \pm 0.04$ & $0.34 \pm 0.12$ & $0.19 \pm 0.07$ \\
low-pass & $0.20 \pm 0.01$ & $0.05 \pm 0.12$ & $0.20 \pm 0.01$ & $0.19 \pm 0.01$ \\
high-pass & $0.20 \pm 0.01$ & $0.00 \pm 0.01$ & $0.20 \pm 0.01$ & $0.20 \pm 0.01$ \\
\hline
\end{tabular}



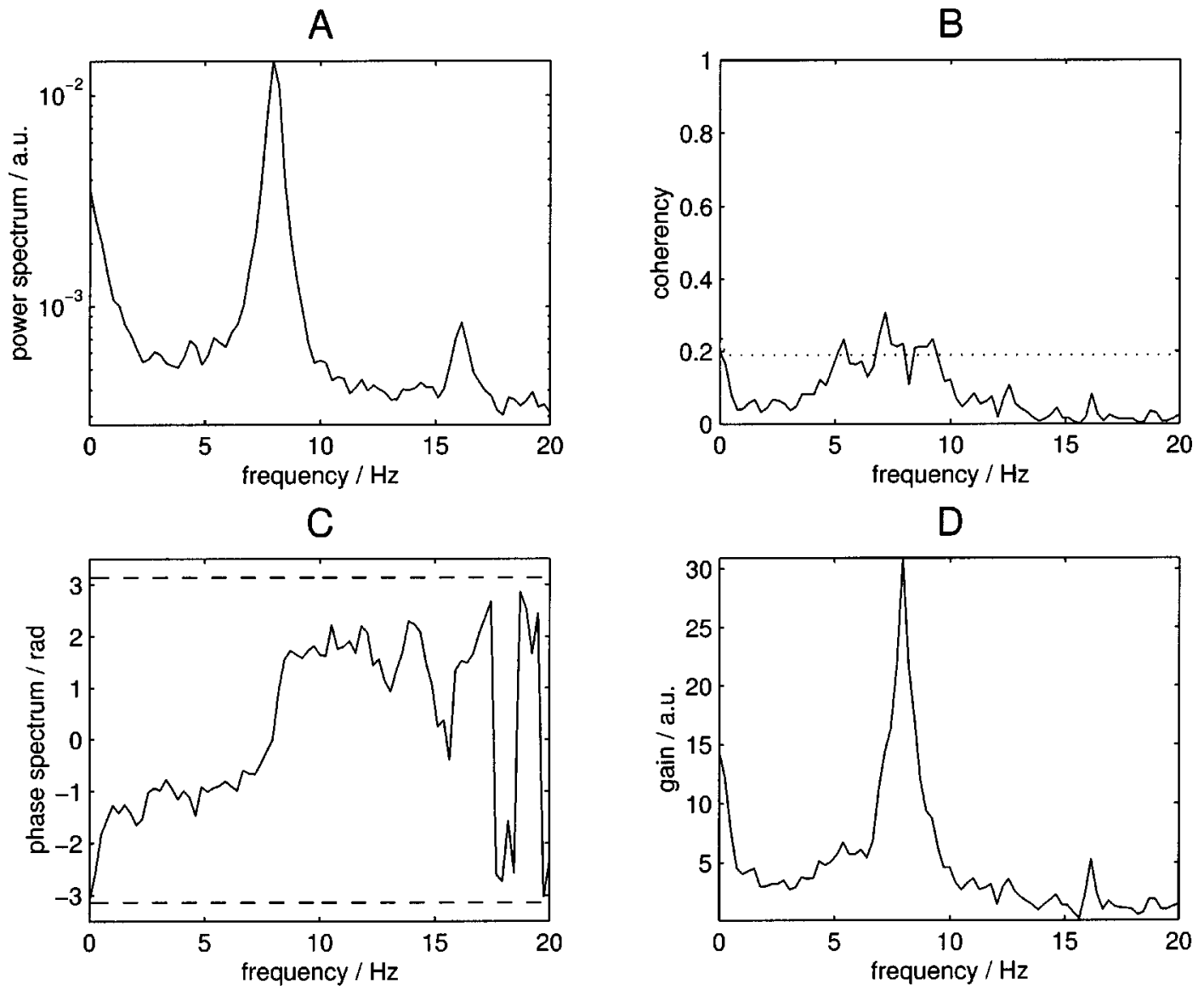

FIGURE 3. Cross-spectral analysis of input and output for one realization of the nonlinear stochastic oscillator. (A) Power spectrum of output; (B) coherency (significance threshold, $\alpha=0.05$, indicated by dotted line); (C) phase spectrum; (D) gain. Noise level, $\mathrm{SNR}_{\text {in }}=\mathrm{SNR}_{\text {out }}=1$. No delay. Parameters: $a_{1}=1.6, a_{2}^{+}=-2.3, a_{2}^{-}=-0.72$, and $s=2.5$.

worst, as indicated both by the bias and by the large variance. That method ignores most of the information available from the phase spectrum. Figure 6 shows the distribution of delay time estimates with the single frequency method for 1000 realizations, $\mathrm{SNR}_{\text {in }}=\mathrm{SNR}_{\text {out }}$ $=1$. The estimates are distributed over a large range and show many outliers. Thus, the single frequency method is inappropriate for delay time estimation in the presence of noise.

In the presence of noise, all four estimators are biased. However, the bias disappears in the noise-free case for the Hilbert transform method, while the three other methods retain a bias (Fig. 5). This bias is due to the fact that the damped stochastic oscillator violates a central assumption underlying all methods except for the Hilbert transform method: the system does not consist of a pure delay only. The oscillator has a nontrivial transfer function whose contribution to the phase spectrum has to be taken into account. This is well achieved by the Hilbert transform method. Figure 7 compares the methods of fitting a straight line to the phase spectrum and the Hilbert transform method. As is to be expected, the correc- tion of the phase spectrum based on Eq. (28) plays a crucial role in removing the bias of the estimator.

The phase spectrum between input $x(t)$ and output $y(t)$ of an $\mathrm{AR}[2]$ process can be calculated analytically. ${ }^{43}$ It exhibits a jump from zero to $\pi$ at the resonance frequency of the AR[2] oscillator:

$$
\Phi(f)=\arctan \left(\frac{a_{1} \sin (2 \pi f)+a_{2} \sin (4 \pi f)}{1-a_{1} \cos (2 \pi f)-a_{2} \cos (4 \pi f)}\right)
$$

The theoretically calculated phase spectrum is shown superimposed on the estimated minimum phase in Fig. 7(D), showing good agreement.

Comparison of Various Model Types. Table 1 summarizes the performance of the four delay estimation procedures using all the five models. The noise level was fixed to $\mathrm{SNR}_{\text {in }}=\mathrm{SNR}_{\text {out }}=1$, and a delay of $0.2 \mathrm{~s}$ was used. For each model, 100 realizations were computed. 
A
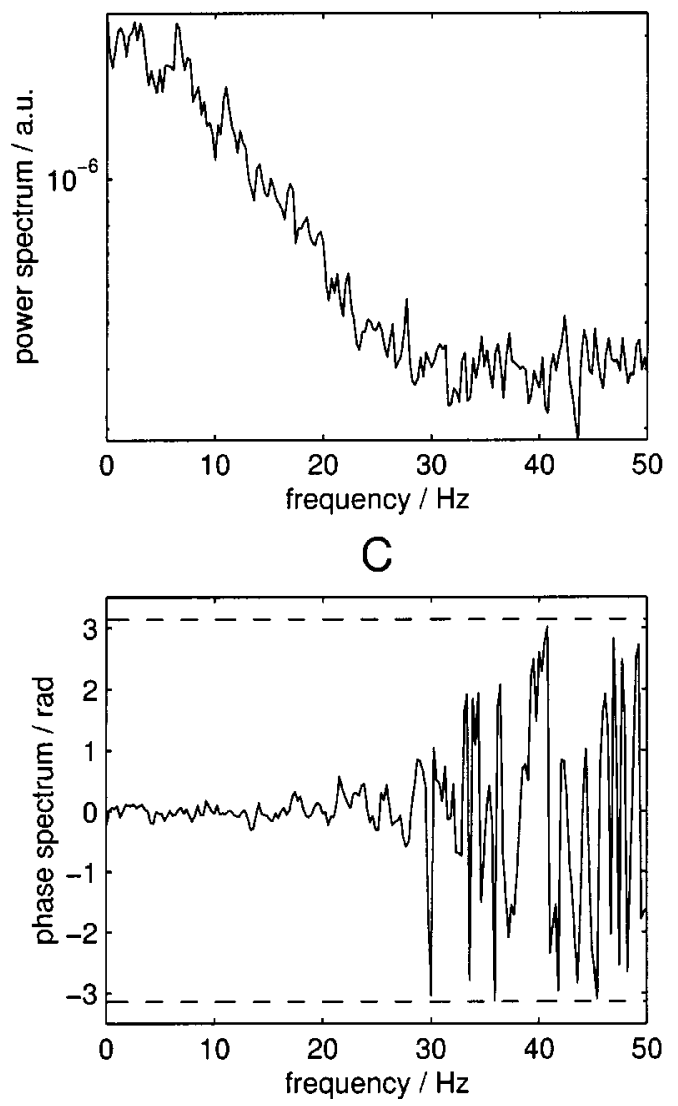

B
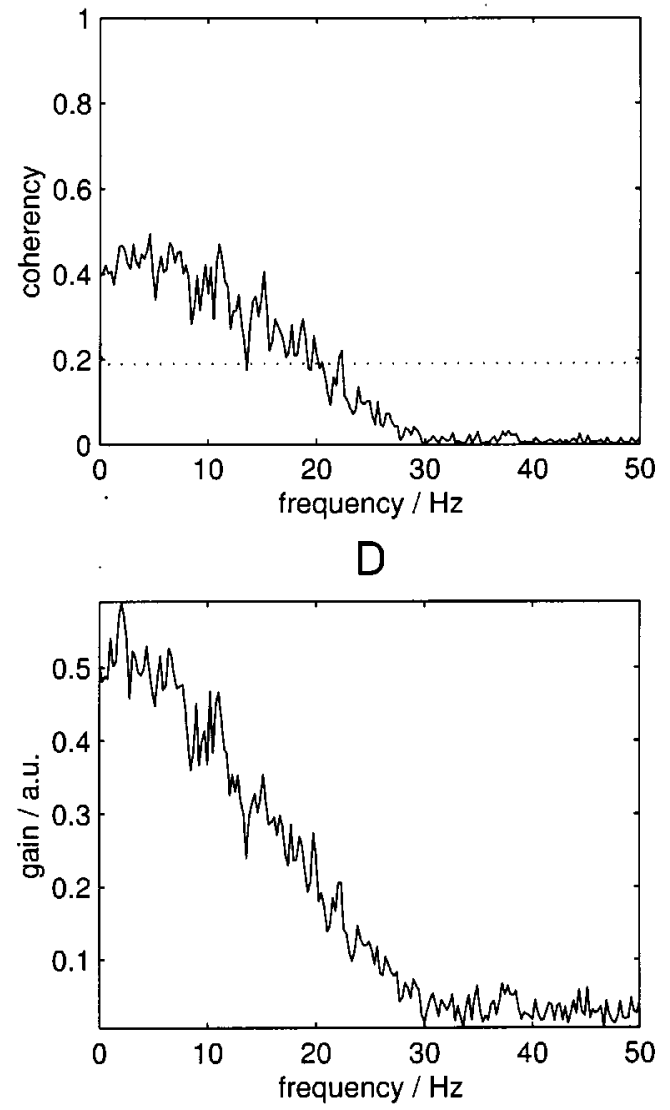

FIGURE 4. Cross-spectral analysis of input and output for one realization of the linear low-pass filter. (A) Power spectrum of output; (B) coherency (significance threshold; $\alpha=0.05$, indicated by dotted line); (C) phase spectrum; (D) gain. Noise level, $S N R_{\text {in }}=S N R_{\text {out }}=1$. No delay. Parameters: $m_{-2}=m_{2}=7 / 96, m_{-1}=m_{1}=1 / 4$, and $m_{0}=17 / 48$.

The table shows that the Hilbert transform method is the most versatile of the four delay estimation methods proposed in this article. Its performance both in terms of bias and of variance is good in four out of five models. An especially encouraging result is its performance in the case of the SETAR[2] model: despite the fact that this model's transfer function is nonlinear, the Hilbert transform method gives an almost unbiased delay time estimate. The relatively poor performance in the case of the stochastic oscillator driven by a nonlinear input (cf. Fig. 2) is due to the fact that the coherency is significant only in a small frequency band around the frequency of the driving force $(\sim 15 \mathrm{~Hz})$, so that in the frequency region where the transfer function of the $\mathrm{AR}[2]$ process has a large effect on the phase (cf. Fig. 1), no data are available.

\section{APPLICATION TO MEASURED DATA}

In this section, we estimate delays in three different physiological systems using measured data from experiments on vestibular stimulation, cerebral autoregulation, and orthostatic tremor.

\section{Vestibular Stimulation}

In this section we apply the Hilbert transform method to a vestibular stimulation experiment described in detail in Ref. 35. In this study, galvanic vestibular stimulation serves to modulate the continuous firing level of the peripheral vestibular afferents. Our objective was to test the hypothesis that stochastic galvanic vestibular stimulation can lead to coherent stochastic postural sway. Bipolar binaural stochastic galvanic vestibular stimulation was applied to healthy young subjects. Three different stochastic vestibular stimulation signals, each with a different frequency content $(0-1,1-2$, and $0-2 \mathrm{~Hz})$, were used. Twenty $60 \mathrm{~s}$ trials were conducted on each subject, 15 stimulation trials (five trials with each stimulation signal) and five control (no stimulation) trials. ${ }^{35}$

Postural sway was evaluated by using a force platform to measure the displacements of the center of pressure (COP) under each subject's feet. We found significant coherency between the stochastic vestibular stimulation signal and the resulting mediolateral COP time series in the majority of trials in eight of the nine subjects tested. ${ }^{35}$ Furthermore, we investigated the delay 

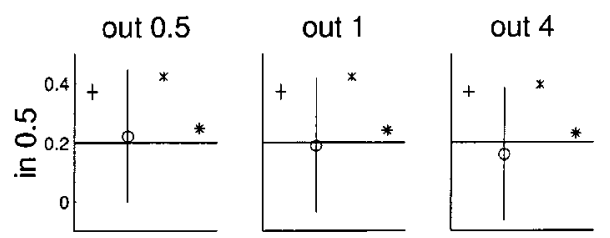

out $\infty$
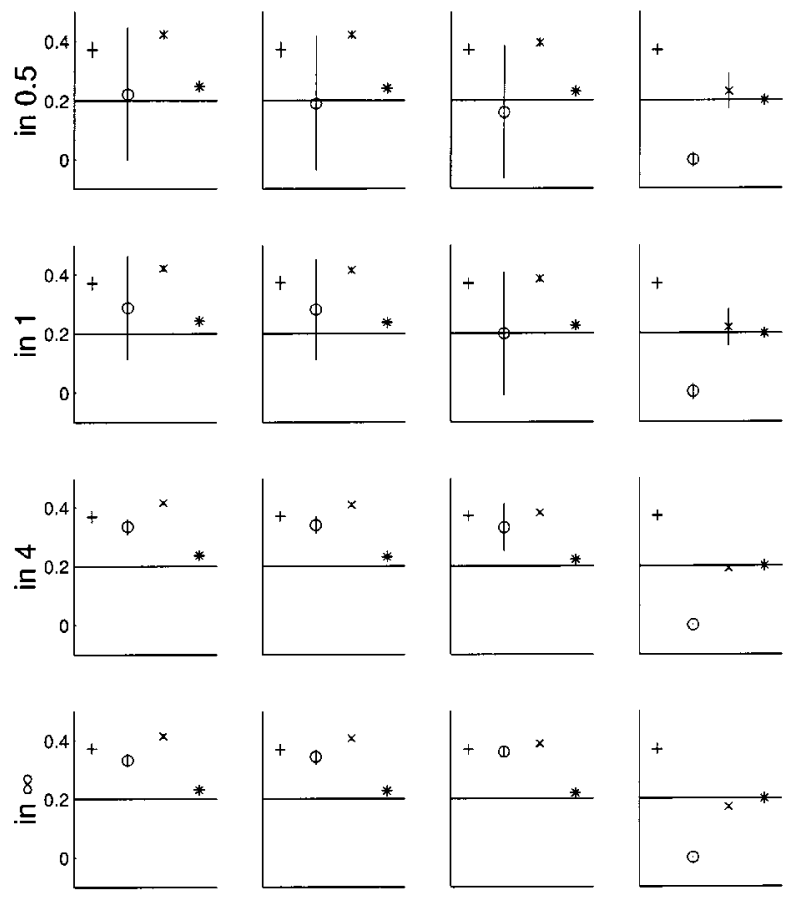

FIGURE 5. Comparison of the four proposed delay estimators for simulated data covered with various levels of observational noise $\left(\mathrm{SNR}_{\text {in }}\right.$ and $\mathrm{SNR}_{\text {out }}$ from 0.5 to infinity). Data were simulated from an AR[2] model [Eq. (31)], parameters: $T=0.8 \mathrm{~s}, \tau=0.8 \mathrm{~s}$. All subplots are on the same scale; the true delay $(0.2 \mathrm{~s})$ is indicated by a solid horizontal line. $\mathrm{Re}$ sults from 100 trials are plotted as mean \pm SD. Symbols: $(+)$ cross correlation method; $(O)$ single frequency method $(X)$ straight line fit to phase spectrum; (*) Hilbert transform method. SD indicated by vertical lines.

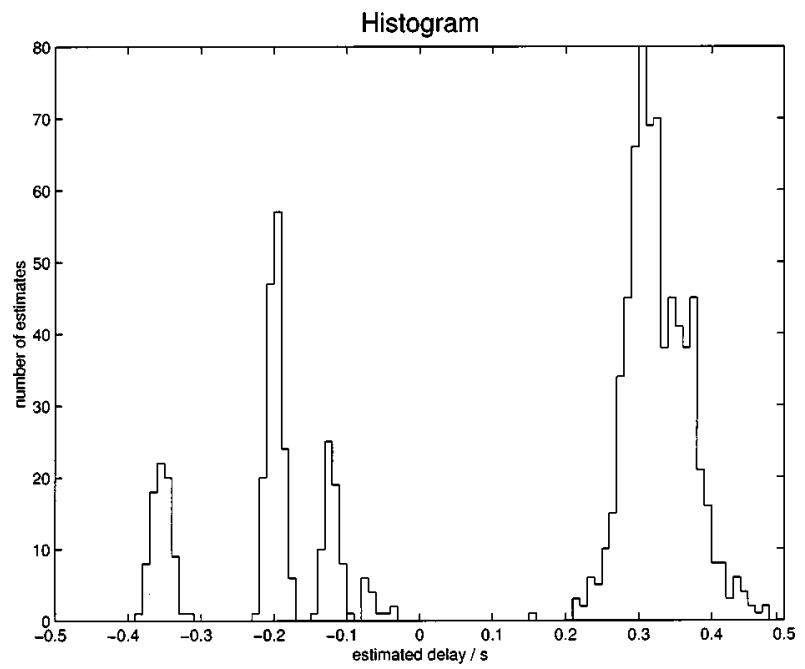

FIGURE 6. Histogram showing the distribution of delay time estimates for the single frequency method, 1000 trials, $S N R_{\text {in }}=S N R_{\text {out }}=1$. True delay, $0.2 \mathrm{~s}$.
A
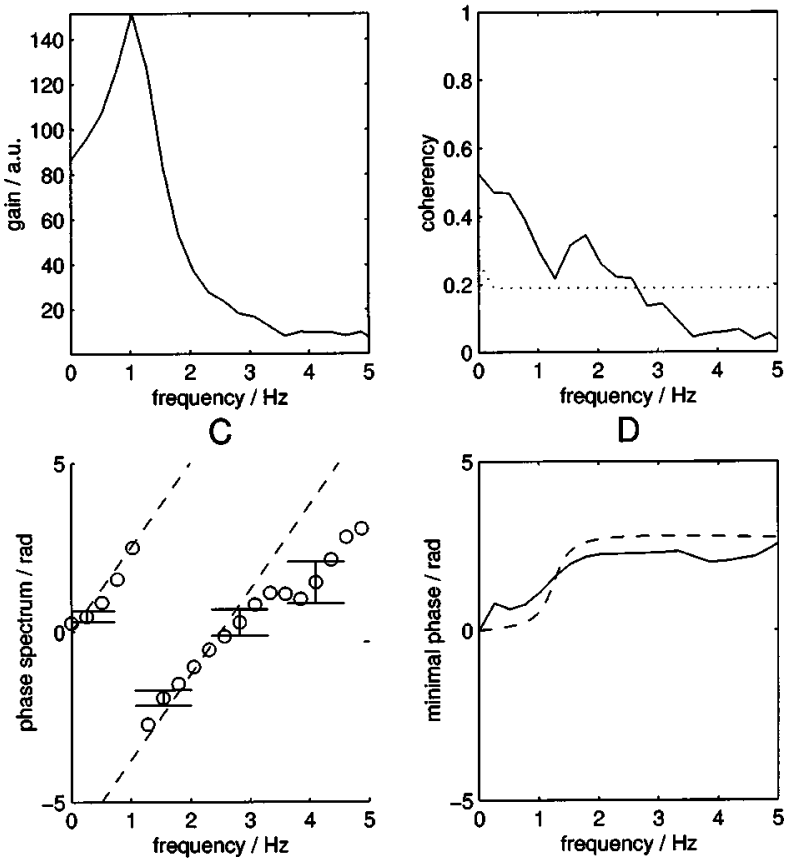

$\mathrm{E}$
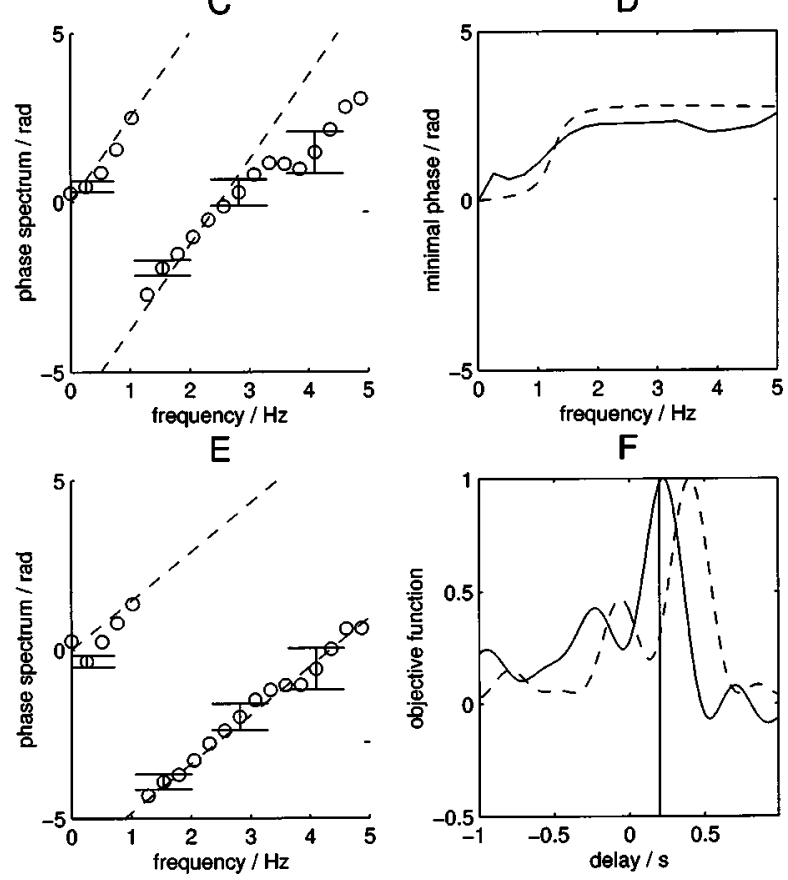

$F$

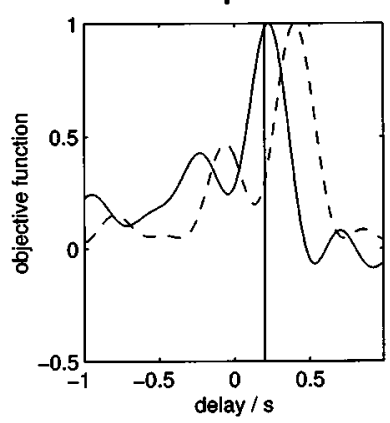

FIGURE 7. Comparison of Hilbert transform method to straight line fit to phase spectrum for a realization of the AR[2] system ( $\left.T=0.8 \mathrm{~s}, \tau=0.8 \mathrm{~s}, \mathrm{SNR}_{\text {in }}=\mathrm{SNR}_{\text {out }}=1\right)$. (A) Gain; (B) coherency (significance threshold, $\alpha=0.05$, indicated by dotted line). (C) Estimated phase spectrum (circles) with best-fit straight line (dashed), corresponding to a delay of $0.4 \mathrm{~s}$. $95 \%$ confidence intervals indicated for every fifth point. (D) Argument of the transfer function estimated via the Hilbert transform method, Eq. (28) (solid line) and true function computed analytically, Eq. (38) (dashed). (E) Corrected phase spectrum (circles) and best-fit straight line (dashed), corresponding to a delay of $0.23 \mathrm{~s}$. Confidence intervals as in (C). (F) Objective function (scaled to maximum 1) for the straight line fit (dashed) and for the Hilbert transform method (solid line). True delay, $0.2 \mathrm{~s}$, indicated by a solid vertical line.

times between the stimulus signal and the coherent postural sway by the delay time estimation procedure that fits a straight line to the phase spectrum, assuming a "delay-only" model.

The estimates reported in Ref. 35 result in significantly different delay times for each of the stimulus signals. The group averages were $1.09 \mathrm{~s}$ for the $0-1 \mathrm{~Hz}$ 
TABLE 2. Delays (in $\mathrm{ms}$ ) for the vestibular stimulation experiment, estimated with the Hilbert transform method. Each number is the average of estimates from at least two trials. (*) Insufficient data. The total average is $522 \pm 127 \mathrm{~ms}$.

\begin{tabular}{cccc}
\hline Subject & $\begin{array}{c}0-1 \mathrm{~Hz} \\
\text { Stimulus }\end{array}$ & $\begin{array}{c}0-2 \mathrm{~Hz} \\
\text { Stimulus }\end{array}$ & $\begin{array}{c}1-2 \mathrm{~Hz} \\
\text { Stimulus }\end{array}$ \\
\hline 1 & 369 & 422 & 359 \\
2 & 622 & 735 & 382 \\
3 & 637 & 639 & 501 \\
4 & 416 & 699 & 387 \\
5 & 679 & $*$ & 429 \\
6 & 539 & 685 & 321 \\
7 & 561 & 679 & 493 \\
8 & 449 & $*$ & 587 \\
9 & 412 & $*$ & 534 \\
Average & 520 & 643 & 444 \\
Std. dev. & 113 & 113 & 89 \\
\hline
\end{tabular}

stimulus, $0.57 \mathrm{~s}$ for the $0-2 \mathrm{~Hz}$ signal, and $0.27 \mathrm{~s}$ for the 1-2 $\mathrm{Hz}$ stimulus.

For physiological reasons it is expected that the delay between stimulus and response be roughly the same (a few hundred $\mathrm{ms}$ ) for all stimuli. If there were really different delay times for different stimulus frequencies, one would have to conclude that different nerve fibers are used to transmit signals of different frequencies, which is physiologically absurd. The frequency dependency observed could be due to filter properties of the system that cause a nontrivial transfer function. The quality of the data is good: the SNR is large (around 10), and each trial is more than 100 times longer than the expected delay. Thus, the Hilbert transform method could be used to correct the phase spectrum for the filter properties of the system.

The results of the Hilbert transform delay time estimation are indeed encouraging. Table 2 reports the results for the nine subjects studied. The average estimated delay is $522 \mathrm{~ms}$, which is in the expected range. While a small frequency dependency of the delay time estimate is still present, it is much less pronounced than with the simple delay estimation method. Figure 8 shows the average gain for the $0-2 \mathrm{~Hz}$ stimulus, subject 3 . The gain shows a high-pass characteristic, resulting in a nontrivial transfer function. Both the simulations and the results of Table 2 suggest that the influence of the filter properties of the system on the phase spectrum can be corrected with the Hilbert transform method.

\section{Analysis of Dynamic Cerebral Autoregulation}

Cerebral autoregulation (CA) keeps the perfusion in the brain relatively constant over a wide range of arterial blood pressure (ABP). Impairment of $\mathrm{CA}$, often linked to carotid artery stenosis, might increase the risk of ischemic stroke. As CA is a concept rather than a physically measurable quantity, it is not easy to assess. ${ }^{34}$ Early

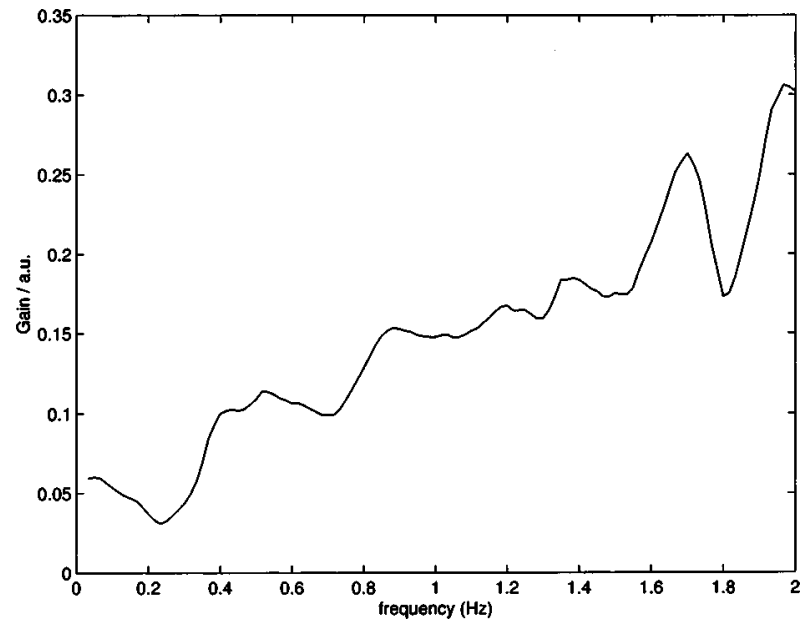

FIGURE 8. Average gain for subject 3 of the vestibular stimulation experiment, $0-2 \mathrm{~Hz}$ stimulus.

measurements of static autoregulation have been superseded by analysis of dynamic CA, following work by Aaslid et al. ${ }^{1}$

Through the advent of transcranial Doppler sonography (TCD), it is possible to measure cerebral blood flow velocity (CBFV) noninvasively with a high temporal resolution. ABP can also be measured noninvasively with the same resolution, using finger plethsymography. The first paradigm for the assessment of dynamic CA was to introduce drops in $\mathrm{ABP}$ via release of thigh cuffs and record the time course of $\mathrm{ABP}$ and $\mathrm{CBFV}$. It has been observed that impaired CA leads to a slower recovery of CBFV following an initial drop, while intact CA leads to fast recovery with a delay reported in the range of $1-2$ s. $^{40}$ Since the thigh cuff technique cannot be used for all patients and tends to be inconvenient, it would be of advantage to use cross-spectral methods to quantify CA from data recorded completely noninvasively. Using this paradigm, a positive phase shift (i.e., CBFV preceding $\mathrm{ABP}$ ) in the low frequency range (around $0.1 \mathrm{~Hz}$ ) has been interpreted as intact CA according to a highpass filter model of the CA feedback control system. ${ }^{13}$ Frequencies of $0.1 \mathrm{~Hz}$ were either obtained by deep breathing or by analyzing spontaneous oscillations of $\mathrm{ABP}$ and $\mathrm{CBFV}$ during supine rest over several minutes. ${ }^{28,34,49}$

We compare data from 18 healthy young volunteers and ten older patients with severe $(>80 \%)$ unilateral stenosis of the internal carotid artery. Time-courses of $\mathrm{ABP}$ (input) and CBFV (output) were recorded over a period of 3-4 min at a sampling frequency of $100 \mathrm{~Hz}$ with the person resting in a supine position and breathing slowly at a rate of $6 / \mathrm{min}(0.1 \mathrm{~Hz})$. These data were analyzed without any further preprocessing.

Figure 9 illustrates the result of delay time estimation for one recording from a healthy subject. Table 3 shows 
A

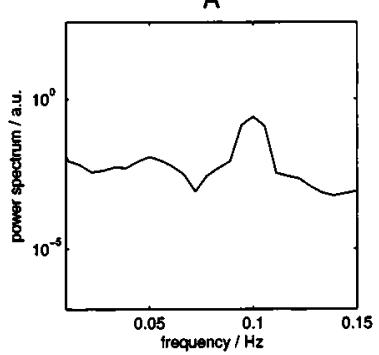

C

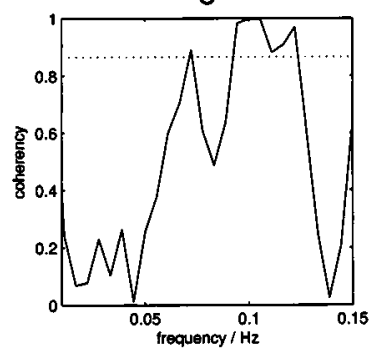

E

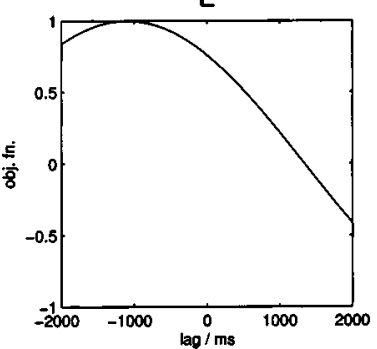

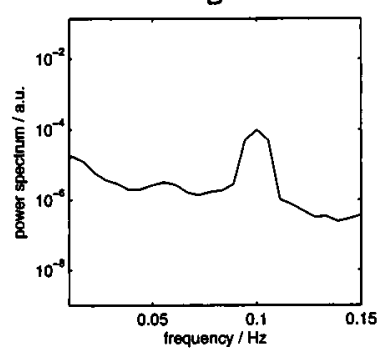

D

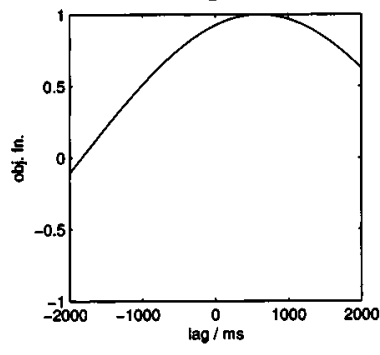

F

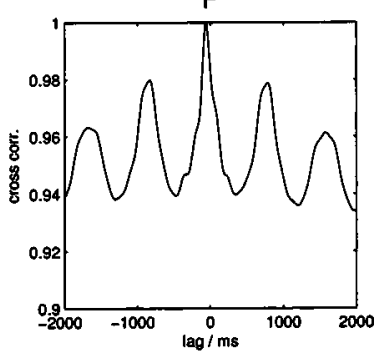

FIGURE 9. Cross-spectral analysis of autoregulation data from right Doppler of a 28-year-old healthy woman. (A) Power spectrum of ABP. (B) Power spectrum of CBFV. (C) Coherency (significance threshold, $\alpha=0.05$, indicated by dotted line). (D) Objective function for the Hilbert transform method. (E) Objective function for the method of fitting a straight line to the phase spectrum. (F) Cross-correlation function.

the results of delay time estimation using the four methods proposed. The cross correlation method yields a small negative delay time around $-60 \mathrm{~ms}$. Both the "single frequency" method which uses the frequency of maximum coherency for each data set to estimate a delay, and the method of fitting a straight line to the phase spectrum yield negative delay times around $-1 \mathrm{~s}$. The standard deviation is higher in the case of the single frequency method. The Hilbert transform method yields positive delay times around $1 \mathrm{~s}$.

Times in the range of $-60 \mathrm{~ms}$, as obtained by the cross correlation method, have been interpreted as delay times in the CA system, ${ }^{9}$ but physiological considerations yield a different interpretation: Each pulse wave originates from the heart, and the pulse wave takes longer to reach the finger, where $\mathrm{ABP}$ is recorded, than it takes to reach the brain, where CBFV is recorded. Indeed, the observed delay computed with the cross correlation method $(-0.056 \pm 0.019 \mathrm{~s}$ for all persons) matches well with the propagation speed of $5 \mathrm{~m} / \mathrm{s}$ for pressure waves in arteries given in the physiological literature. ${ }^{39}$ Thus, the cross correlation method does not yield the sought-for delay time of the CA system.

As changes in $\mathrm{ABP}$ cause changes in $\mathrm{CBFV}$, a physiological delay time in the CA system between $\mathrm{ABP}$ as input and CBFV as output should be positive. It is to be expected that the phase spectrum obtained through spectral analysis, which is used both in the single frequency method and in the method of fitting a straight line to the phase spectrum, contains information from a delay time and from the transfer function of the CA system, since the CA system acts as a high-pass filter. ${ }^{13}$ Thus, the negative delay times obtained by the two methods mentioned can be interpreted as physiological delay times, as observed using a protocol of stepwise ABP changes, covered by the effect of the high-pass filter transfer function. The higher variance in the case of the single frequency method is due to the fact that the method of fitting a straight line to the phase spectrum uses more information from the phase spectrum. The method of fitting a straight line to the phase spectrum differentiated clearly between between young normal subjects and patients' affected sides $(-0.56 \mathrm{~s}$ vs. $-1.38 \mathrm{~s}$, respectively; $\mathrm{t}$ test, $p$ $<0.01$ ) and between patients' affected and contralateral sides (paired Wilcoxon-test, $p=0.014$ ).

The Hilbert transform method yields positive delays in the range of $1 \mathrm{~s}$, which are both physiologically meaningful in view of the causal relationship between ABP and $\mathrm{CBFV}$ and are similar to times observed via step changes in ABP. Thus, it seems that the Hilbert transform

TABLE 3. Results of delay time estimation (in s) for the cerebral autoregulation data. Insufficiently coherent recordings (less than four significantly coherent frequencies in the range of $0.05-0.14 \mathrm{~Hz}$ ) were excluded.

\begin{tabular}{rccc}
\hline & $\begin{array}{c}\text { Healthy volunteers } \\
n=25\end{array}$ & $\begin{array}{c}\text { Patients, affected } \\
n=10\end{array}$ & $\begin{array}{c}\text { Patients, contralateral } \\
n=10\end{array}$ \\
\hline Max. CC & $-0.06 \pm 0.01$ & $-0.03 \pm 0.02$ & $-0.07 \pm 0.02$ \\
Single freq. & $-1.36 \pm 0.47$ & $-0.63 \pm 0.43$ & $-1.23 \pm 0.51$ \\
Line fit & $-1.38 \pm 0.46$ & $-0.56 \pm 0.37$ & $-1.20 \pm 0.48$ \\
Hilbert meth. & $0.76 \pm 0.54$ & $1.25 \pm 0.54$ & $0.79 \pm 0.68$ \\
\hline
\end{tabular}


TABLE 4. Results of delay time estimation (absolute values in ms) for left/right muscle pairs from the orthostatic tremor data. TA, tibalis anterior (calf); TS, triceps surae (calf); L5, trunk muscle at lumbal level (back).

\begin{tabular}{rcccccc}
\hline & $\begin{array}{r}\text { Patient No. 1: TA } \\
n=4\end{array}$ & $\begin{array}{c}\text { No. 1: TS } \\
n=4\end{array}$ & $\begin{array}{c}\text { No. 1: L5 } \\
n=4\end{array}$ & $\begin{array}{c}\text { No. 2: TA } \\
n=4\end{array}$ & $\begin{array}{c}\text { No. 2: TS } \\
n=4\end{array}$ & $\begin{array}{c}\text { No. 2: L5 } \\
n=4\end{array}$ \\
\hline Max. CC & $3 \pm 0$ & $4 \pm 1$ & $0 \pm 0$ & $1 \pm 0$ & $3 \pm 1$ & $0 \pm 0$ \\
Single freq. & $2 \pm 1$ & $5 \pm 1$ & $0 \pm 0$ & $2 \pm 1$ & $3 \pm 2$ & $6 \pm 1$ \\
Line fit & $3 \pm 0$ & $5 \pm 1$ & $1 \pm 1$ & $2 \pm 1$ & $4 \pm 2$ & $5 \pm 1$ \\
Hi bert meth. & $4 \pm 1$ & $4 \pm 1$ & $0 \pm 1$ & $4 \pm 2$ & $3 \pm 2$ & $6 \pm 2$ \\
\hline
\end{tabular}

method can correct for the high-pass filter properties of the CA system, yielding delay times as observed with the invasive thigh cuff technique by analyzing oscillations recorded noninvasively. The Hilbert transform method is also able to distinguish patients from normal subjects: for the young normal subjects, the estimated delay time is $0.76 \mathrm{~s}$, while the patients' values on the affected side $(1.25 \mathrm{~s})$ are significantly different (t test, $p=0.02)$. Within the group of patients, the delay on the affected side differed significantly from the delay on the contralateral side (paired Wilcoxon-test, $p=0.049$ ).

Thus, the Hilbert transform method appears to be a promising tool for the assessment of cerebral autoregulation.

\section{Orthostatic Tremor}

Orthostatic tremor (OT) is a relatively rare form of human tremor that is mainly observed in orthostasis in affected patients. The main characteristics of OT are its high frequency of oscillations of over $15 \mathrm{~Hz}$ as compared to roughly $5-12 \mathrm{~Hz}$ for other tremor forms and, most prominently, the fact that affected muscles all over the body exhibit highly correlated electromyogram (EMG) recordings. ${ }^{27,29}$

There has been some debate on the question of the location of a generator for OT. ${ }^{27}$ The site of the oscillation source can presumably be identified through comparison of delay times between EMG signals from a number of muscles. If the hypothesis is correct that the OT generator is located supraspinally, e.g., in the cortex, it should be possible to explain estimated delay times between two muscles as the difference of the delays between cortex and first muscle and between cortex and second muscle. If the generator is not located in the cortex, or if there is no single oscillator responsible for the observed highly coherent oscillations, the proposed explanation of estimated delays should not be possible. We investigate which of the four proposed delay time estimators can be employed to answer the question of the location of an OT generator.

EMGs of various muscles were recorded from two patients affected by OT. Each recording session was $20 \mathrm{~s}$ long (sampling rate, $3 \mathrm{kHz}$; standard preprocessing according to Ref. 23). Six EMGs could be recorded simul- taneously. Seven sessions were recorded for patient No. 1, eight sessions for No. 2. We focus on three muscle pairs: left/right tibalis anterior (TA; calf), left/right triceps surae (TS; calf), and left/right trunk muscles at the lumbal level (L5; back). Physiological considerations predict the following: ${ }^{24}$ (1) The delay between left and right muscles of the same sort should be zero. (2) The muscles TA and TS are an agonist-antagonist pair, meaning that only one of them is activated at any time. Thus, an oscillation in the calf should consist of one half period of activation of TA, followed by one half period of activation of TS. Accordingly, the delay between TA and TS should be equal to one half tremor period $(\pi$ radians). By (1), this should hold no matter whether TA and TS are recorded on the same side of the body or on different sides. An acceptable delay time estimation method for the explanation of OT data should at least fulfill conditions (1) and (2).

Table 4 presents the results for all four delay time estimators with respect to condition (1). Table 5 presents the results for condition (2). Figure 10 shows as an example the results of cross-spectral analysis of an EMG recording of right TA vs. TS from patient No. 1. Patient No. 1 has a tremor frequency of roughly $18 \mathrm{~Hz}$. The power spectra for TA and TS are quite similar, showing the fundamental frequency and one higher harmonic at which the signals are significantly coherent. The second higher harmonic is also present to a lesser extent [cf. Fig. 10(C)]. Patient No. 2 has a tremor frequency of roughly

TABLE 5. Results of delay time estimation (absolute values in ms) for agonist-antagonist calf muscle pair tibalis anterior (TA) and triceps surae (TS) in the orthostatic tremor data. Abbreviation of methods as in Table 1. The last line gives the time of half a tremor period, which is the physiologically expected delay time.

\begin{tabular}{rcc}
\hline & $\begin{array}{c}\text { Patient No. 1: TA vs. TS } \\
n=22\end{array}$ & $\begin{array}{c}\text { No. 2: TA vs. TS } \\
n=22\end{array}$ \\
\hline Max. CC & $23 \pm 4$ & $24 \pm 9$ \\
Single freq. & $21 \pm 2$ & $4 \pm 5$ \\
Line fit & $24 \pm 6$ & $23 \pm 11$ \\
Hi bert meth. & $27 \pm 3$ & $25 \pm 8$ \\
Half period & 28 & 33 \\
\hline
\end{tabular}


A

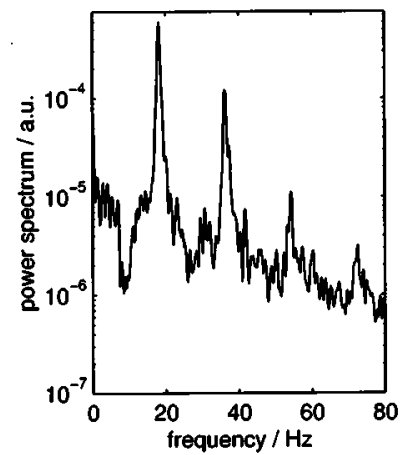

C

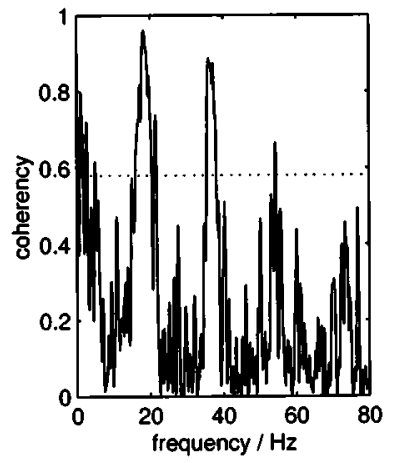

E

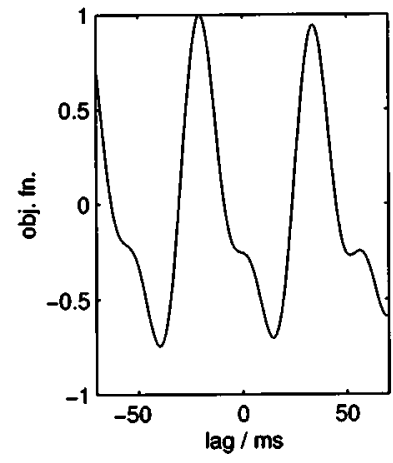

B

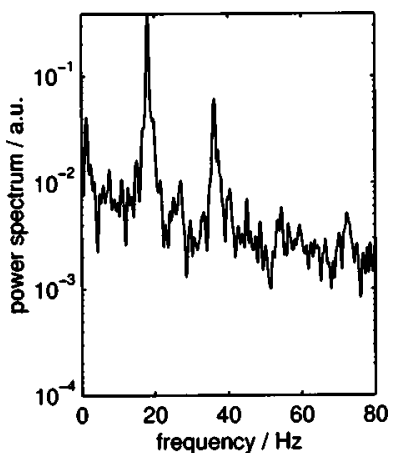

D

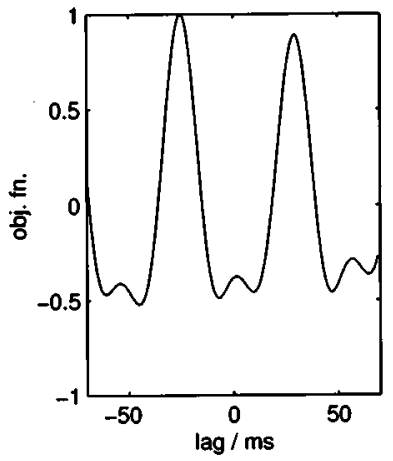

$F$

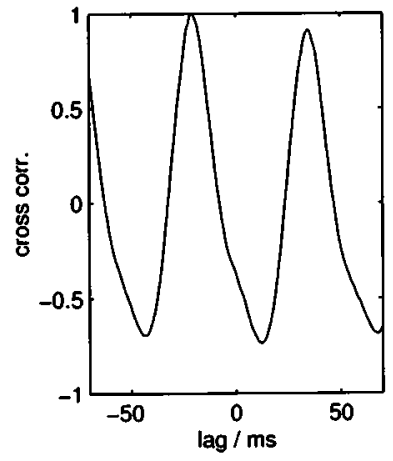

FIGURE 10. Cross-spectral analysis of orthostatic tremor data from patient No. 1, right calf muscle tibalis anterior (TA) vs. right calf muscle triceps surae (TS). (A) Power spectrum of the TA electromyogram; (B) same for TS; (C) coherency (significance threshold, $\alpha=0.05$, indicated by dotted line); (D) objective function for the Hilbert transform method. (E) Objective function for the method of fitting a straight line to the phase spectrum. (F) Cross correlation function.

$15 \mathrm{~Hz}$. The signals show high coherency up to the fourth higher harmonic (not shown).

Figure 10 also shows the objective functions for the Hilbert transform method [Eq. (29); Fig. 10(D)] and for the method of fitting a straight line to the phase spectrum [Eq. (26); Fig. 10(E)] as well as the cross correlation function [Fig. 10(F)]. These objective functions are nearly periodic with a period of one tremor oscillation (56 ms).
Table 4 shows that all four methods performed quite well as regards condition (1). There is hardly any difference in estimated delay times. The consistent small positive delay time between left/right muscle pairs might suggest that the tremor source is located off the body middle axis, but no physiological conclusions can be drawn at this stage. With respect to condition (2), Table 5 shows that all four methods performed quite well for patient No. 1, with the Hilbert transform method yielding the estimate closest to the expected delay time and a small variance. The data from patient No. 2 posed problems for all methods. The single frequency method performed badly. Again, the Hilbert transform method came closest to the expected value and had a smaller variance than its competitors. Since signal transmission in nerves is affected by the filter properties of the neural pathways, the results of Table 5 suggest that the Hilbert transform method is able to correct for the neural filter properties, even though the effect is not tremendous. In further applications to orthostatic tremor data, delay times should thus be estimated by the Hilbert transform method.

\section{DISCUSSION}

Four delay estimators were compared with the focus on practical applicability of methods. The crosscorrelation method takes the lag at which the cross correlation between input and output is maximal as a delay time. The other three methods are cross-spectral methods based on an interpretation of the phase spectrum between input and output. The simplest procedure interprets the phase at the single frequency of maximum coherency directly in terms of a delay. More generally, one can fit a straight line to the phase spectrum in order to estimate a delay time. The Hilbert transform method subtracts the effect of a nontrivial transfer function from the phase spectrum before fitting a straight line.

Through simulation studies the following image emerged: In the presence of noise, all four estimators showed a bias. The bias tended to be smallest for the Hilbert transform method for most model types. In the presence of a nonlinear transfer function, the Hilbert transform method gave good results. Thus, even though cross-spectral analysis is a concept designed for linear transfer functions, it yields helpful information in the nonlinear case, too, provided that input and output are significantly coherent (i.e., can be explained to a certain extent by a linear model) at some frequencies.

The Hilbert transform method contains as a limiting case the method of fitting a straight line to the phase spectrum. That method is always superior to the pointwise interpretation of the phase spectrum - the variance is lower, and often the bias is smaller as well. Thus, a pointwise interpretation of the phase spectrum should never be used to estimate a delay time. The method of 
fitting a straight line to the phase spectrum yields good results if a "delay only" model holds, but it is susceptible to a large bias if that assumption is violated. There are certainly applications for which the cross-correlation method performs best. However, apart from the fact that the Hilbert transform method can correct for a nontrivial transfer function in the system, one can also select the band of frequencies to be used for the fit, while the cross-correlation method, operating in the time domain, offers no such possibility easily.

A notable exception to the good performance of the Hilbert transfer method was the case of the oscillator driven by a nonlinear input. The poor performance of the method in this case could be explained by the fact that input and output were only significantly coherent at the frequency of the driving force, whereas the nontrivial transfer function that the Hilbert transform method should correct for, operated at frequencies at which input and output were not coherent (cf. Fig. 2). Thus, the prospects of the Hilbert transform method are best when signals are coherent over a large frequency region.

The application of the four delay estimators to measured data confirmed the results of the simulations. For the vestibular stimulation data, the method of fitting a straight line to the phase spectrum had led to frequencydependent delay times that were not easy to reconcile with the physiology of the experiment. The Hilbert transform method led to nearly constant delay times for all stimulus frequencies, thus confirming the influence of a nontrivial transfer function (cf. Fig. 8) on the delay time estimate. For the cerebral autoregulation data, the crosscorrelation method yielded results that could be interpreted physiologically, but were not related to the sought-for delay time of the autoregulation system. Both the pointwise interpretation of the phase spectrum and the method of fitting a straight line to the phase spectrum yielded negative delay times, while the Hilbert transform method, again correcting for a nontrivial transfer function, led to results that could be interpreted as a physiologically meaningful delay time. Furthermore, these delay times were significantly different in patients on the side of carotid artery stenosis as compared to the contralateral side or to controls, suggesting that the delay time estimated via the Hilbert transform method could be used to assess the functional state of the cerebral autoregulatory system. Finally, for the orthostatic tremor data all four proposed methods except for the single frequency method yielded sensible results, but the Hilbert transform method was somewhat better able to explain the agonist-antagonist relationship of the calf muscles tibalis anterior and triceps surae than the other methods. This result suggests that in further applications of delay time estimation for orthostatic tremor data, the Hilbert transform method should be the method of choice. It must be conceded that in the orthostatic tremor data, the case for the Hilbert transform method is not as strong as in the other two applications, but the method still performed better than its competitors.

In summary, we have shown that the Hilbert transform method is a valuable general-purpose nonparametric tool for delay time estimation that can be applied fruitfully to empirical data.

\section{ACKNOWLEDGMENTS}

T.M. and M.R. acknowledge support from the German Federal Ministry of Education and Research $(b m b+f)$. The authors thank B. Guschlbauer, B. Hellwig, and B. Schelter for help with the orthostatic tremor data and J. J. Collins and J. T. Inglis for help with the vestibular stimulation data. We would also like to thank three anonymous reviewers for their detailed comments on a previous version of this article and for helpful suggestions.

\section{REFERENCES}

${ }^{1}$ Aaslid, R., K. F. Lindegaard, W. Sorteberg, and H. Nornes. Cerebral autoregulation dynamics in humans. Stroke 20:4552, 1989.

${ }^{2}$ Avitzour, D. Time delay estimation at high signal-to-noise ratio. IEEE Trans. Aerosp. Electron. Syst. 27:234-237, 1991.

${ }^{3}$ Azenkot, Y., and I. Gertner. The least squares estimation of time delay between two signals with unknown relative phase shift. IEEE Trans. Acoust., Speech, Signal Process. 33:308309, 1985.

${ }^{4}$ Bloomfield, P. Fourier Analysis of Time Series: An Introduction. New York: Wiley, 1976, p. 225.

${ }^{5}$ Brockwell, P. J., and R. A. Davis. Time Series: Theory and Methods. New York: Springer, 1991, p. 434.

${ }^{6}$ Cabot, R. C. A note on the application of the Hilbert transform to time delay estimation. IEEE Trans. Acoust., Speech, Signal Process. 29:607-609, 1981.

${ }^{7}$ Chan, Y. T., R. V. Hattin, and J. B. Plant. The least squares estimation of time delay and its use in signal detection. IEEE Trans. Acoust., Speech, Signal Process. 26:217-222, 1978.

${ }^{8}$ Chan, Y. T., and R. K. Miskowicz. Estimation of coherence and time delay with ARMA models. IEEE Trans. Acoust., Speech, Signal Process. 32:295-303, 1984.

${ }^{9}$ Chiu, C.-C., and S.-Y. Yeh. Assessment of cerebral autoregulation using time-domain cross-correlation analysis. Comput. Biol. Med. 31:471-480, 2001.

${ }^{10}$ Cleveland, W. S., and E. Parzen. The estimation of coherence, frequency response, and envelope delay. Technometrics 17:167-172, 1975.

${ }^{11}$ Clifford, C. G. Coherence and time delay estimation. Proc. IEEE 75:236-255, 1987.

${ }^{12}$ Deaton, M. L., and R. V. Foutz. Group delay and the timelag relationship between stochastic processes. J. Time Ser. Anal. 1:111-118, 1980

${ }^{13}$ Diehl, R. R., D. Linden, D. Lücke, and P. Berlit. Phase relationship between cerebral blood flow velocity and blood pressure. A clinical test of autoregulation. Stroke 26:18011804, 1995.

${ }^{14}$ Hamon, B. V., and E. J. Hannan. Spectral estimation of time 
delay for dispersive and nondispersive systems. Appl. Stat. 23:134-142, 1974.

${ }^{15}$ Hannan, E. J., and P. J. Thomson. The estimation of coherence and group delay. Biometrika 58:469-481, 1971.

${ }^{16}$ Hannan, E. J., and P. J. Thomson. Estimating group delay. Biometrika 60:241-253, 1973.

${ }^{17}$ Hannan, E. J., and P. J. Thomson. Delay estimation and the estimation of coherence and phase. IEEE Trans. Acoust., Speech, Signal Process. 29:485-490, 1981.

${ }^{18}$ Hannan, E. J., and P. J. Thomson. Time delay estimation. J. Time Ser. Anal. 9:21-33, 1988.

${ }^{19}$ Hertz, D., and M. Azaria. Time delay estimation between two phase shifted signals via generalized cross-correlation methods. Signal Process. 8:237-255, 1985.

${ }^{20}$ Hinich, M. J., and G. R. Wilson. Time delay estimation using the cross bispectrum. IEEE Trans. Signal Process. 40:106113, 1992.

${ }^{21}$ Holm, S., and G. Ottesen. Bias in the cross spectrum and time delay estimates due to misalignment. IEEE Trans. Acoust., Speech, Signal Process. 34:1662-1665, 1986.

${ }^{22}$ Honerkamp, J. Stochastic Dynamical Systems. New York: VCH, 1994.

${ }^{23}$ Journee, H. L. Demodulation of amplitude modulated noise: a mathematical evaluation of a demodulator for pathological tremor EMG's. IEEE Trans. Biomed. Eng. 30:304-308, 1983.

${ }^{24}$ Kloeden, P. E., E. Platen, and S. H. The numerical solution of nonlinear stochastic dynamical systems: A brief introduction. Int. J. Bif. Chaos 1:277-286, 1991.

${ }^{25}$ Knapp, C. H., and G. C. Carter. The generalized correlation method for estimation of time delay. IEEE Trans. Acoust., Speech, Signal Process. 24:320-327, 1976.

${ }^{26}$ Köster, B., M. Lauk, J. Timmer, M. Poersch, B. Guschlbauer, G. Deuschl, and C. H. Lücking. Involvement of cranial muscles and high intermuscular coherence in orthostatic tremor. Ann. Neurol. 45:384-388, 1999.

${ }^{27}$ Köster, B., M. Lauk, J. Timmer, M. Poersch, B. Guschlbauer, G. Deuschl, and C. H. Lücking. Involvement of cranial muscles and high intermuscular coherence in orthostatic tremor. Ann. Neurol. 45:384-388, 1999.

${ }^{28}$ Kuo, T. B., C. M. Chern, W. Y. Sheng, W. J. Wong, and H. $\mathrm{H}$. Hu. Frequency domain analysis of cerebral blood flow velocity and its correlation with arterial blood pressure. $J$. Cereb. Blood Flow Metab. 18:311-318, 1998.

${ }^{29}$ Lauk, M., B. Köster, J. Timmer, B. Guschlbauer, G. Deuschl, and C. H. Lücking. Side-to-side correlation of muscle activity in physiological and pathological human tremor. Clin. Neurophysiol. 110:1774-1783, 1999.

${ }^{30}$ Lindemann, M., J. Raethjen, J. Timmer, G. Deuschl, and G. Pfister. Delay estimation for cortico-peripheral relations. $J$. Neurosci. Methods 111:127-139, 2001.

${ }^{31}$ Nakano, J., and S. Tagami. Delay estimation by a Hilbert transform method. Austrl. J. Statist. 30:217-227, 1988.

${ }^{32}$ Nikias, C. L., and R. Pan. Time delay estimation in unknown Gaussian spatially correlated noise. IEEE Trans. Acoust., Speech, Signal Process. 36:1706-1714, 1988.

${ }^{33}$ Oppenheim, A. V., and R. W. Schafer. Digital Signal Processing. London: Prentice-Hall, 1975.

${ }^{34}$ Panerai, R. B., R. P. White, H. S. Markus, and D. H. Evans. Grading of cerebral dynamic autoregulation from spontaneous fluctuations in arterial blood pressure. Stroke 29:23412346, 1998

${ }^{35}$ Pavlik, A. E., J. T. Inglis, M. Lauk, L. Oddsson, and J. J. Collins. The effects of stochastic galvanic vestibular stimulation on human postural sway. Exp. Brain Res. 124:273-280, 1999.

${ }^{36}$ Piersol, A. G. Time delay estimation using phase data. IEEE Trans. Acoust., Speech, Signal Process. 29:471-477, 1981.

${ }^{37}$ Press, W., B. Flannery, S. Saul, and W. Vetterling. Numerical Recipes, 2nd ed. London: Cambridge University Press, 1992.

${ }^{38}$ Priestley, M. Spectral Analysis and Time Series. New York: Academic, 1989.

${ }^{39}$ Schmidt, R. F., and G. Thews. Human Physiology, 2nd ed. Berlin: Springer, 1989.

${ }^{40}$ Tiecks, F. P., A. M. Lam, R. Aaslid, and D. W. Newell. Comparison of static and dynamic cerebral autoregulation measurements. Stroke 26:1014-1019, 1995.

${ }^{41}$ Timmer, J. Parameter estimation in nonlinear stochastic differential equations. Chaos, Solitons Fractals 11:2571-2578, 2000.

${ }^{42}$ Timmer, J., M. Lauk, and G. Deuschl. Quantitative analysis of tremor time series. Electroencephalogr. Clin. Neurophysiol. 101:461-468, 1996.

${ }^{43}$ Timmer, J., M. Lauk, W. Pfleger, and G. Deuschl. Crossspectral analysis of physiological tremor and muscle activity. I. Theory and application to unsychronized EMG. Biol. Cybern. 78:349-357, 1998.

${ }^{44}$ Tong, H. Threshold Models in Nonlinear Time Series Analysis, Vol. 21 of Lecture Notes in Statistics. New York: Springer, 1983.

${ }^{45}$ Tribolet, J. M. A new phase unwrapping algorithm. IEEE Trans. Acoust., Speech, Signal Process. 25:170-177, 1977.

${ }^{46}$ van der Pol, B. On oscillation-hysteresis in a simple triode generator. Philos. Mag. 43:177, 1922.

${ }^{47}$ Youn, D. H., and N. Ahmed. Time delay estimation via coherence: An adaptive approach. J. Acoust. Soc. Am. 75:505514, 1984.

${ }^{48}$ Youn, D. H., N. Ahmed, and G. C. Carter. An adaptive approach for time delay estimation of band-limited signals. IEEE Trans. Acoust., Speech, Signal Process. 31:780-784, 1983.

${ }^{49}$ Zhang, R., J. H. Zuckerman, C. A. Giller, and B. D. Levine. Transfer function analysis of dynamic cerebral autoregulation in humans. Am. J. Physiol. 274:H233-H241, 1998. 